\title{
Optical trapping and manipulation of nanostructures
}

\author{
Onofrio M. Maragò ${ }^{1 \star}$, Philip H. Jones², Pietro G. Gucciardi', Giovanni Volpe ${ }^{3}$ and Andrea C. Ferrari ${ }^{4 \star}$
}

\begin{abstract}
Optical trapping and manipulation of micrometre-sized particles was first reported in 1970 . Since then, it has been successfully implemented in two size ranges: the subnanometre scale, where light-matter mechanical coupling enables cooling of atoms, ions and molecules, and the micrometre scale, where the momentum transfer resulting from light scattering allows manipulation of microscopic objects such as cells. But it has been difficult to apply these techniques to the intermediate - nanoscale range that includes structures such as quantum dots, nanowires, nanotubes, graphene and two-dimensional crystals, all of crucial importance for nanomaterials-based applications. Recently, however, several new approaches have been developed and demonstrated for trapping plasmonic nanoparticles, semiconductor nanowires and carbon nanostructures. Here we review the state-of-the-art in optical trapping at the nanoscale, with an emphasis on some of the most promising advances, such as controlled manipulation and assembly of individual and multiple nanostructures, force measurement with femtonewton resolution, and biosensors.
\end{abstract}

ight can exert a force on matter by means of momentum exchange on scattering ${ }^{1}$. The existence of this force was first experimentally demonstrated by Lebedev ${ }^{2}$ and Nichols and $\mathrm{Hull}^{3}$ in 1901 using thermal light sources (electric or arc lamps) and a torsion balance. When the light was focused on a mirror attached to the balance, the radiation pressure moved the balance from its equilibrium position ${ }^{2,3}$. But the magnitude of these effects was considered insignificant for any practical use: to quote J. H. Poynting's presidential address to the British Physical Society in 1905 (reported in ref. 4), "A very short experience in attempting to measure these forces is sufficient to make one realize their extreme minuteness a minuteness which appears to put them beyond consideration in terrestrial affairs." It was not until 1970, and because of the advent of lasers, that Arthur Ashkin showed that the use of optical forces to alter the motion of micrometre-sized particles ${ }^{5}$ and neutral atoms ${ }^{6}$ could have applications in the manipulation of microscopic particles and of single atoms ${ }^{4}$.

These pioneering works have developed into two very successful research lines. On one hand, early techniques for laser cooling of atoms ${ }^{7-10}$ paved the way to modern ultracold atom technology ${ }^{11}$. On the other hand, what is now commonly referred to as optical tweezers - that is, a tightly focused laser beam capable of confining particles in three dimensions ${ }^{12}$ - has become a common tool for the manipulation of micrometre-sized particles ${ }^{13,14}$ and as a highly sensitive force transducer ${ }^{15}$. But optical forces acting between $\sim 1$ and $100 \mathrm{~nm}$, a range of primary interest for nanotechnology (Fig. 1), have not been widely exploited because of the challenges in scaling up the techniques optimized for atom cooling, or scaling down those used for microparticle trapping. Indeed, efficient laser cooling of atoms relies on light scattering close to a narrow spectral line, without radiative losses, to reduce the atomic velocity distribution ${ }^{11}$. Nanostructures lack these features, limiting both the cooling rate and the minimum achievable temperature ${ }^{11}$. The techniques used for manipulating microparticles rely on the electric dipole interaction energy ${ }^{16,17}$. Because this scales down approximately with the particle volume, thermal fluctuations are large enough to overwhelm the trapping forces at the nanoscale ${ }^{18}$.

New approaches were thus developed to stably trap and manipulate nanoparticles. Over the past few years, these techniques have been successfully applied to a variety of objects, for example metal nanoparticles (MNPs $)^{19-21}$, plasmonic nanoparticles $(\mathrm{NPs})^{22-30}$, quantum $\operatorname{dots}^{31,32}$, carbon nanotubes $(\mathrm{CNTs})^{33-37}$, graphene flakes ${ }^{38,39}$, nanodiamonds $s^{40}$, polymer nanofibres ${ }^{41}$ and semiconductor nanowires ${ }^{42-49}$. Typically these techniques rely either on special properties of the trapped objects themselves - for example force enhancement related to plasmonic resonances supported by the trapped particles ${ }^{22-30}$, or highly anisotropic geometries, such as in CNTs and nanowires ${ }^{35,38,43,44,46,47}$ - or on new approaches to optical manipulation, such as exploiting the field enhancement due to plasmons supported by nanostructures on a substrate ${ }^{50-57}$, or the feedback on the optical forces of the trapped object $^{58}$. Optical manipulation has been used to build composite nanoassemblies ${ }^{32,42,43,59}$. Optical tweezers have been developed to measure forces with femtonewton resolution, enabling the study of interactions between nanoobjects ${ }^{34,59-64}$. They have also been integrated with spectroscopic techniques, such as Raman spectroscopy ${ }^{33,36,38,65-71}$ and photoluminescence $e^{40,44,45,48,49,72}$, paving the way to the selection and manipulation of NPs after their individual characterization ${ }^{36,38}$. Optically levitated nanoparticles have been laser-cooled towards their quantum-mechanical ground state $\mathrm{g}^{73-76}$.

Here, we review the state-of-the-art, open questions and future directions in optical trapping and manipulation of nanostructures, and show how the development of these techniques can affect nanoscience and nanotechnology.

\section{Optical forces on nanostructures}

In this section, we review how optical forces arise. We first consider the case of particles much smaller than the trapping wavelength where one can make use of the Rayleigh approximation. We then address the case of larger particles, where the full electromagnetic scattering theory must be employed. We finally discuss plasmonenhanced forces and optical binding, particularly relevant for optical trapping and manipulation of nanostructures.

Forces in the dipole approximation. The optical response of a nanostructure can be often modelled as that of a dipole ${ }^{16}$ or a collection of dipoles ${ }^{17}$. The dipolar polarizability determines the

${ }^{1}$ CNR-IPCF, Istituto per i Processi Chimico-Fisici, I-98158 Messina, Italy, ${ }^{2}$ Department of Physics and Astronomy, University College London, London WC1E 6BT, UK, ${ }^{3}$ Department of Physics, Bilkent University, Cankaya, Ankara 06800, Turkey, ${ }^{4}$ Cambridge Graphene Centre, University of Cambridge, $9 \mathrm{JJ}$ Thomson Avenue, Cambridge CB3 OFA, UK.

*e-mail: marago@me.cnr.it; acf26@hermes.cam.ac.uk 
strength of interaction with an optical field ${ }^{16}$. For a sphere of radius $a$ and relative permittivity $\varepsilon$, this can be written $\operatorname{as}^{77}$ :

$$
\alpha=\frac{\alpha_{0}}{1-\frac{i k^{3} \alpha_{0}}{6 \pi \varepsilon_{0}}}
$$

where $\alpha_{0}$ is the point-like particle polarizability given by the Clausius-Mossotti relation ${ }^{77} \alpha_{0}=4 \pi \varepsilon_{0} a^{3}(\varepsilon-1) /(\varepsilon+2) ; k$ is the field wavevector; and $\varepsilon_{0}$ is the vacuum dielectric permittivity. The denominator in equation (1) acts as a correction to the ClausiusMossotti relation to account for the reaction of a finite-sized dipole to the scattered field at its own location ${ }^{77}$. The time-averaged force acting on such a dipole is ${ }^{16}$ :

$$
\langle\mathbf{F}\rangle=\frac{1}{2} \operatorname{Re}\left(\sum_{j=x, y, z} \alpha E_{j} \nabla E_{j}^{*}\right)
$$

where $E_{j}$ are the electric field components. Equation (2) can be recast into the more explicit form ${ }^{78}$ :

$$
\langle\mathbf{F}\rangle=\underbrace{\frac{1}{4} \operatorname{Re}(\alpha) \nabla|\mathbf{E}|^{2}}_{\text {Intensity gradient }}+\underbrace{\frac{\sigma}{2 c} \operatorname{Re}\left(\mathbf{E} \times \mathbf{H}^{*}\right)}_{\text {Radiation pressure }}+\underbrace{\frac{\sigma c \varepsilon_{0}}{4 \omega i} \nabla \times \mathbf{E} \times \mathbf{E}^{*}}_{\text {Polarization gradient }}
$$

where $\sigma$ is the extinction cross-section, $\mathbf{E}$ the electric field, $\mathbf{H}$ the magnetic field, $c$ the speed of light in vacuum, and $\omega$ the angular frequency of the optical field. The first term in equation (3) is the force due to the gradient of the electric field intensity, which permits three-dimensional confinement in optical tweezers ${ }^{12}$ as long as it dominates the second and third terms. The second term, responsible for the radiation pressure, corresponds to a force in the propagation direction ${ }^{5}$. The third term is a force arising from the presence of spatial polarization gradients ${ }^{78}$.
Forces beyond the dipole approximation. When a particle cannot be approximated as a dipole, for example in the case of CNTs, nanowires, graphene and other two-dimensional material flakes, the time-averaged radiation force $F_{\text {rad }}$ on the centre of mass due to scattering of an electromagnetic field is equal in magnitude, and opposite in sign, to the rate of change of momentum of the electromagnetic field itself ${ }^{79-84}$. Therefore, $\mathbf{F}_{\text {rad }}$ can be calculated by integrating the optical momentum flux over a closed orientable surface $S$ surrounding the object ${ }^{81,83}$ :

$$
\mathbf{F}_{\mathrm{rad}}=\int_{S}\left\langle\mathbf{T}_{\mathrm{M}}\right\rangle \cdot \mathrm{d} \mathbf{S}
$$

where $\mathbf{T}_{M}$ is the Maxwell stress tensor, accounting for the interaction between electromagnetic forces and mechanical momentum ${ }^{79,80}$, which can be calculated from the scattered fields, and $\mathrm{d} \mathbf{S}$ is an outward-directed element of surface area. The time-averaged radiation torque $\Gamma_{\text {rad }}$ on the centre of mass can be calculated in an analogous way as ${ }^{85}$ :

$$
\Gamma_{\mathrm{rad}}=-\int_{S}\left\langle\mathbf{T}_{\mathrm{M}}\right\rangle \times \mathbf{r} \cdot \mathrm{d} \mathbf{S}
$$

where $\mathbf{r}$ is the position of the element of surface area.

The scattered electromagnetic fields in equations (4) and (5) can be calculated using Maxwell's equations. Often, however, this turns out to be a cumbersome procedure ${ }^{79}$. Various algorithms have therefore been developed to handle this ${ }^{79}$. In the transition-matrix (T-matrix) method ${ }^{82-89}$, the total electromagnetic field - that is, the sum of incident and scattered field outside the particle and the field internal to the particle - is calculated by expanding all fields in a common orthogonal basis set of functions and imposing boundary conditions on the object surface ${ }^{82,84,86,87}$. Most often, the T-matrix method uses vector spherical wavefunctions ${ }^{79}$ to take advantage of the spherical symmetry of the scatterer, for example Au or polymer NPs ${ }^{84,88}$. Because the T-matrix works best with objects highly

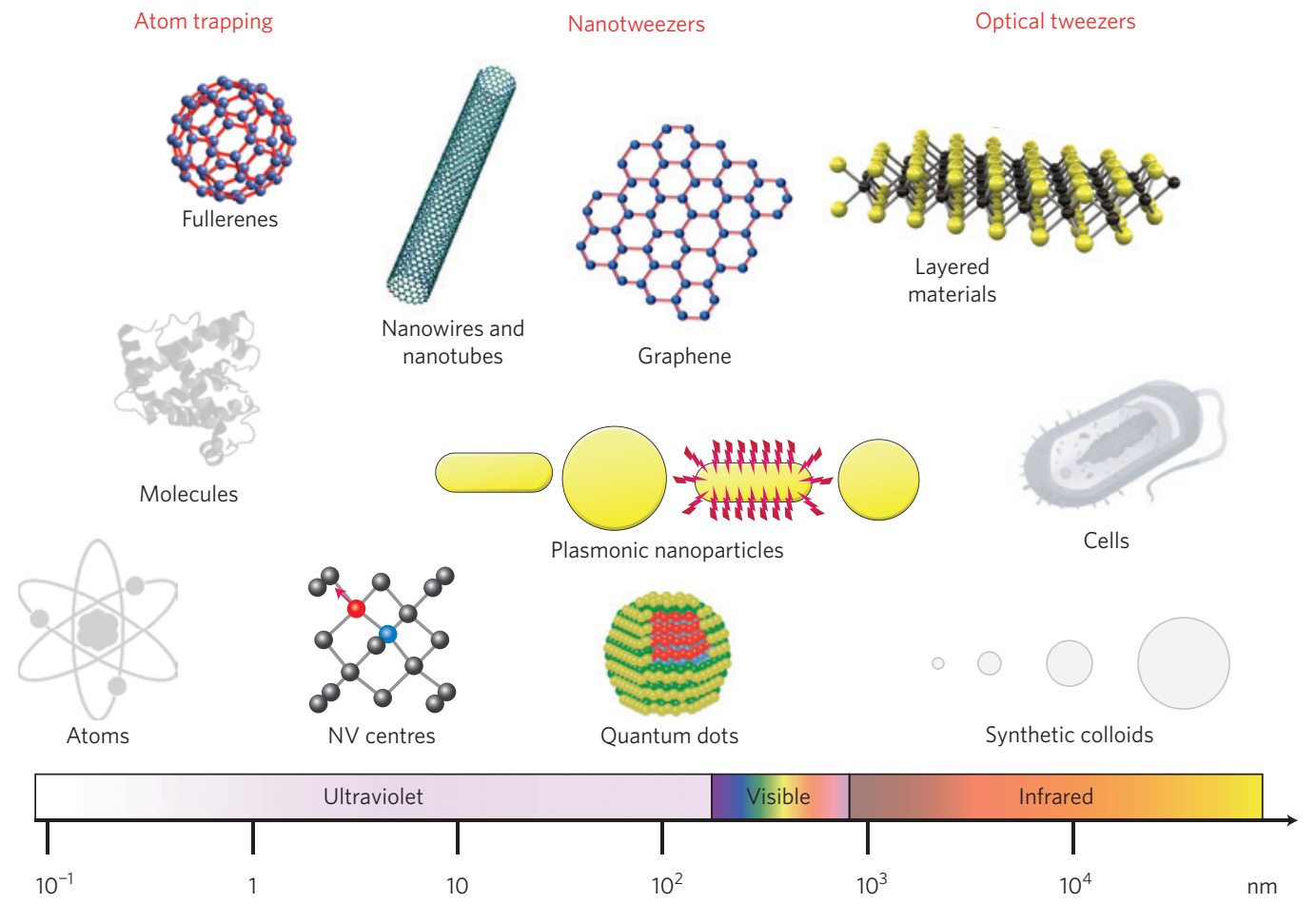

Figure 1 | The three size ranges of optical trapping. Objects of different sizes can be trapped within three main regimes (from left to right): atom trapping (a few ångströms to a few nanometres), nanotweezers (a few nanometres to a few hundred nanometres) and optical tweezers (from a fraction of a micrometre up). The horizontal scale bar shows the average object size and the corresponding light wavelength. NV, nitrogen vacancy. Image of layered material reproduced from ref. 169, (c) 2011 NPG. 
symmetric in shape and composition, one can treat non-spherical objects by modelling them as clusters of small spheres ${ }^{29,41,89}$.

Another method to calculate scattered electromagnetic fields is the discrete dipole approximation $(\mathrm{DDA})^{90}$, also referred to as the coupled dipole model $(\mathrm{CDM})^{17}$, where the particle is modelled as a collection of dipoles. The force on each dipole is due to the incident field and the fields scattered by all other dipoles (equation (2)). The force acting on the particle is given by the sum of the forces acting on each dipole. The torque on the particle can be calculated in an analogous way. The DDA, although more computationally intensive than T-matrix ${ }^{91}$, can be directly applied to particles of any shape and composition. Hybrid methods ${ }^{92,93}$ have also been developed that make use of the T-matrix obtained by pointmatching the near-fields calculated with DDA to get the radiation force and torque.

Plasmon-enhanced forces. Two main approaches can be exploited to use plasmons to enhance optical forces on nanoparticles. The first, discussed in this section, is to use the plasmons supported by trapped MNPs to enhance their mechanical reaction to the fields ${ }^{22-30}$. The second, covered in the section 'Plasmonic tweezers', uses plasmons supported by nanostructures on a substrate, for example pads ${ }^{52,53}$, nanoantennas ${ }^{5,55}$ and nanoholes ${ }^{5,58}$, to generate enhanced fields, in which nanoparticles can be more effectively trapped ${ }^{50}$.

The optical gradient forces (the first term in equation (3)) experienced by nanoparticles are typically very weak (some femtonewtons or less), because the dipolar polarizability given by equation (1) scales with the third power of the particle size ${ }^{77}$. The volume-scaling of the maximum trapping force was evaluated explicitly in ref. 94 for polystyrene spheres, showing a decrease of three orders of magnitude in the maximum trapping force as the sphere radius decreased from 100 to $10 \mathrm{~nm}$. Therefore, to confine nanoparticles against the destabilizing effects of thermal fluctuations, a significantly higher optical power is required: whereas a micrometre-sized polystyrene sphere can be stably trapped with a fraction of a milliwatt in a standard optical tweezers set-up (Fig. 2a,b), a 100-nm sphere requires $15 \mathrm{~mW}$ (ref. 12). This implies that for a $10-\mathrm{nm}$ sphere $\sim 1.5 \mathrm{~W}$ would be needed. The plasmonic nature ${ }^{95}$ of MNPs can enhance the optical forces, so that stable trapping can be achieved at a much lower power ( 2-3 mW; refs 26,29,71). On the one hand, far from plasmon resonances the optical response of small $(<100-\mathrm{nm})$ spherical MNPs is (mainly) the optical response of the free-electron plasma ${ }^{95}$ yielding a large near-infrared (NIR) polarizability ${ }^{95}$. On the other hand, MNPs are resonant systems ${ }^{95}$ and their optical properties (polarizability, cross-sections) are regulated by plasmon resonances that can be tuned by changing size, shapes or aggregation ${ }^{95}$.

Svoboda and Block ${ }^{19}$ compared 36.2-nm Au spheres with 38-nm polystyrene ones, finding a maximum trapping force nearly seven times as great for Au spheres, as a result of the (seven times) greater polarizability at the 1,064-nm trapping wavelength ${ }^{19}$. Both Au nanoparticles (AuNPs, diameters 9.5-254 nm) ${ }^{20}$ and Ag nanoparticles (AgNPs, diameters 20-275 $\mathrm{nm})^{21}$ have been optically trapped in three dimensions. In both cases a maximum trapping force proportional to the third power of the particle radius was observed for diameters $<100 \mathrm{~nm}$, with a crossover to a lower exponent for larger radiii ${ }^{20,21}$. This size-scaling behaviour was interpreted by accounting for local heating ${ }^{96,97}$ of the surroundings, and modelling the MNPs as enclosed in a small steam bubble ${ }^{88,29}$.

Non-spherical MNPs, including Au nanorods (NRs) 22,24 (that is, nanocylinders with an aspect ratio $<10), \mathrm{Ag}$ nanowires ${ }^{98}$ and aggregates of $\mathrm{AuNPs}^{29}$, can sustain plasmon resonances in a broad spectral region in the visible/NIR. These play a crucial role in the enhancement of radiation forces and torques in optical tweezers $^{22-24,26-29}$. More specifically, elongated plasmonic nanostructures (such as nanowires and NRs) are usually trapped with their axis parallel to the electric field vector of the trapping laser, and orthogonal to the propagation axis ${ }^{22,24,84}$. The strength of this aligning torque is increased by tuning the laser close to the plasmon resonance ${ }^{22}$. This provides a means to control their orientation by rotating the laser polarization $^{26}$. Plasmonic nanostructures with lengths from tens of nanometres to several micrometres were aligned and rotated using a single beam of linearly polarized NIR light ${ }^{27}$. Dienerowitz et al..$^{25}$ drew on elements of atom trapping ${ }^{11}$, changing the sign of the gradient force by blue-detuning the laser wavelength with respect to the MNP plasmon resonance. Thus, particle confinement was achieved in the dark spot of an optical vortex beam. The frequency dependence of the plasmon-enhanced radiation force was also used in a system of two counterpropagating evanescent waves at different wavelengths to selectively guide MNPs of different sizes in opposite directions ${ }^{30}$. Cylindrical vector beams with radial polarization were also suggested to trap plasmonic NPs, because for these structured beams the second term in equation (3) (that is, the radiation pressure that pushes particles out of the trap) is zero on the beam axis ${ }^{99}$. Such structured beams trapped both dielectric microparticles ${ }^{100,101}$ and single-walled nanotubes (SWNTs) ${ }^{102}$. Further analysis of the optical forces ${ }^{103}$ revealed, however, that in this case the polarization gradient contribution to the optical force (the third term in equation (3)) can be significant ${ }^{103}$ and may eliminate the advantage of such structured beams.

Resonant illumination of plasmonic NPs gives rise to strong heating effects because of light absorption ${ }^{104}$. Temperature increases of hundreds of kelvin were observed by trapping AuNPs adjacent to fluorophore-containing lipid vesicles with permeability sensitive to temperature ${ }^{97}$. When heated above the gel-transition temperature, fluorophores diffused out of the vesicle $e^{97}$. Further experiments made use of the differing longitudinal and transverse plasmon resonances of AuNRs to control the local heating through the orientation of the AuNRs with respect to the electric field vector of the trapping laser ${ }^{105}$. It was suggested ${ }^{105}$ that this would make AuNRs sensitive and switchable remote-controlled heat transducers to small-volume samples ${ }^{105}$.

Optical binding forces. Optical binding forces emerge from multiple scattering between several objects, and can result in the formation of regular, ordered structures ${ }^{106-108}$. This offers a path towards large-scale NP assembly and organization in one $\mathrm{e}^{109}$, two ${ }^{110}$ and three dimensions ${ }^{111}$. For example, one-dimensional chains of MNPs were suggested as an 'optical sail'112 to achieve a high driving force on an attached nanoscopic object, taking advantage of the huge extinction cross-section of the collective plasmon resonance. Pairs of 200-nm AuNPs were optically bound perpendicular to the direction of light propagation in an optical 'line trap' formed by reflection of a line-shaped focused beam, with particle separations multiples of the optical wavelength ${ }^{109}$, consistent with predictions based on light scattering from Rayleigh (dipolar) particles ${ }^{113}$. Yan et al. ${ }^{110}$ used 40-nm-diameter AgNPs, a size well within the dipole approximation, with both a line trap and a cylindrically symmetric Bessel beam trap, and observed dimers, chains and 'photonic clusters' 110 .

Optical binding interactions can also trap and organize onedimensional carbon nanostructures. In Fig. 2c, we show SWNT bundles illuminated in aqueous suspension ${ }^{114}$ by counterpropagating evanescent fields formed by total internal reflection at a glass/ water interface. We observe their self-organization into optically bound chains, where the bundle axes align parallel to the chain axis and to the direction of propagation of the incident beams. These chains break as soon as the evanescent field is switched off.

\section{Experimental designs and techniques}

In this section, we review the most common experimental implementations relevant for optical tweezers, with an emphasis on those used for trapping and manipulation of NPs and nanostructures. 


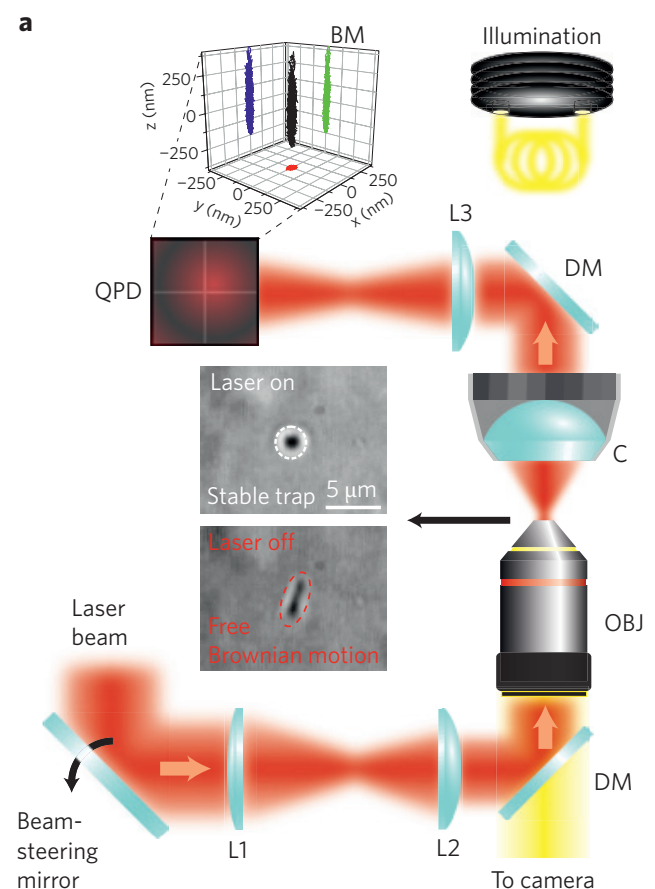

b

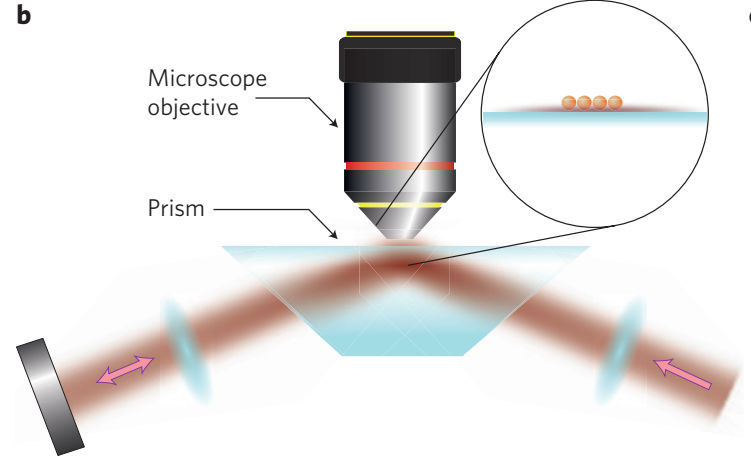

d

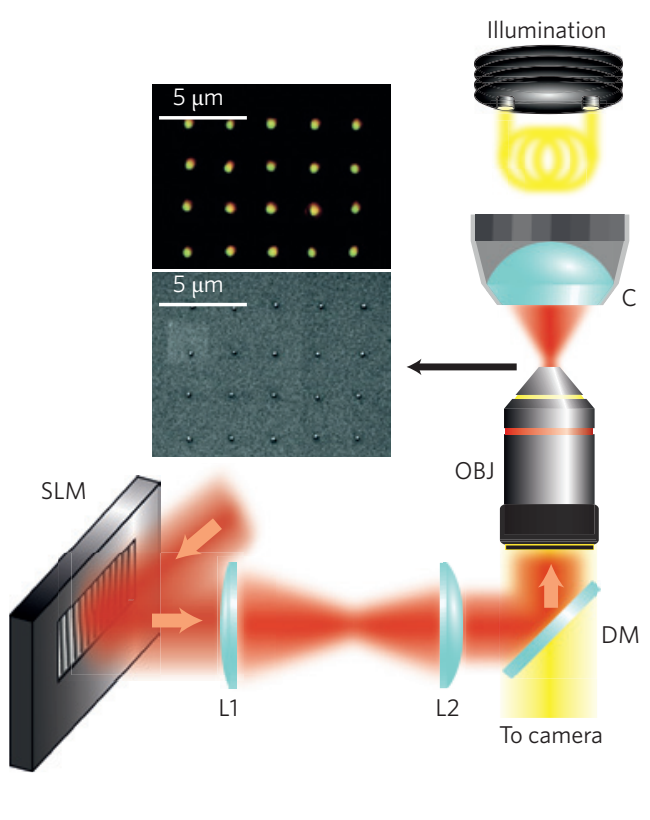

c
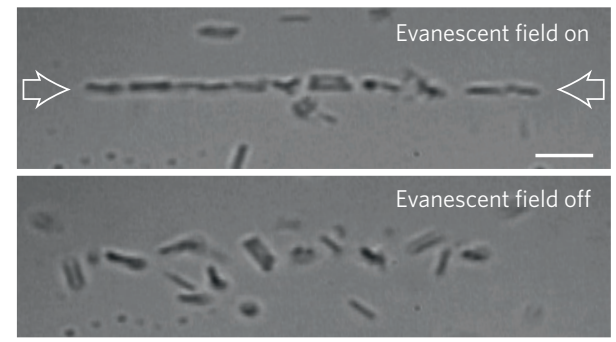

Figure 2 | Basic experimental designs. a, Optical tweezers are obtained by focusing a laser beam to a diffraction-limited spot, using a high-numericalaperture objective lens (OBJ). Additional optics is needed to steer the optical tweezer position (beam-steering mirror and telescope formed by lenses L1, L2), to image the sample (illumination, dichroic mirrors DM, and camera) and to track it (condenser, lens L3 and quadrant photodiode QPD). The resulting traces allow tracking of the Brownian motion (BM) and the calibration of the optical tweezer stiffness. Inset: Bright-field image of (top) optically trapped and (bottom) free SWNT bundle. $\mathbf{b}$, Evanescent optical waves can be excited by total internal reflection at an interface between a high- and a low-refractiveindex medium, often a glass-water interface. The excited evanescent waves can be used to manipulate dielectric and metallic particles. A microscope objective images the sample. c, The optical forces resulting from a standing evanescent wave created by the interference of two counterpropagating evanescent fields (arrows) align SWNT bundles end-to-end (top). When the evanescent wave is switched off, the bundles are released from the locked position and undergo thermal motion (bottom). Scale bar, $5 \mu \mathrm{m}$. d, HOTs rely on a programmable diffractive optical element (SLM) for the creation, shaping and control of multiple independent optical tweezers. Inset: (top) dark-field and (bottom) scanning electron microscope images of a $5 \times 5$ pattern of 80 -nm AuNPs deposited by HOTs on a glass substrate. Figure reproduced with permission from: a, ref. 35, (c) 2008 ACS: inset in d, ref. 144 , (c) 2011 ACS.

Optical tweezers. In the simplest configuration (Fig. 2a), optical tweezers can be generated by focusing a laser beam to a diffraction-limited spot using a high numerical aperture (NA) objective lens $s^{4,115,116}$. This serves the dual purpose of focusing the trapping light and imaging the trapped object. Samples are often placed in small (few microlitres) microfluidic chambers held on a motorized or piezo-driven microscope stage with nanometre position resolution $^{116}$. Generally, optical tweezers require little power (down to a few milliwatts ${ }^{15,116}$ ): carbon and silicon nanostructures have been trapped with as little as $1-2 \mathrm{~mW}$ NIR light ${ }^{34,35,38,46,117}$. The optical tweezer position can be controlled using two steerable mirrors ${ }^{118,119}$. It is also possible to generate multiple optical tweezers by deflecting a single beam in various positions using, for example, an acoustooptic deflector - that is, a device where intensity and frequency of an acoustic wave spatially controls the optical beam ${ }^{115,120}$.
Holographic optical tweezers. The range of optical tweezer applications has been greatly expanded by the use of advanced beam-shaping techniques, where the shape of a light beam is altered by diffractive optical elements (DOEs) to produce multiple optical traps at definite positions ${ }^{13,14}$. Figure $2 \mathrm{~d}$ shows a schematic of a holographic optical tweezer (HOT) set-up, where the DOE is placed in a plane conjugate to the objective focal plane so that the complex field distribution in the trapping plane is the Fourier transform of that in the DOE plane $e^{121,122}$. Often the DOE is a liquid-crystal spatial light modulator (SLM) used to modulate the phase of the incoming beam, because any modulation of the amplitude of the beam would entail a loss of optical power ${ }^{121,122}$. Therefore, various techniques have been developed to determine the optimal phase modulation, for example the Gerchberg-Saxton algorithm, based on iterative optimization of the phase profile at the SLM in order to obtain the desired optical 

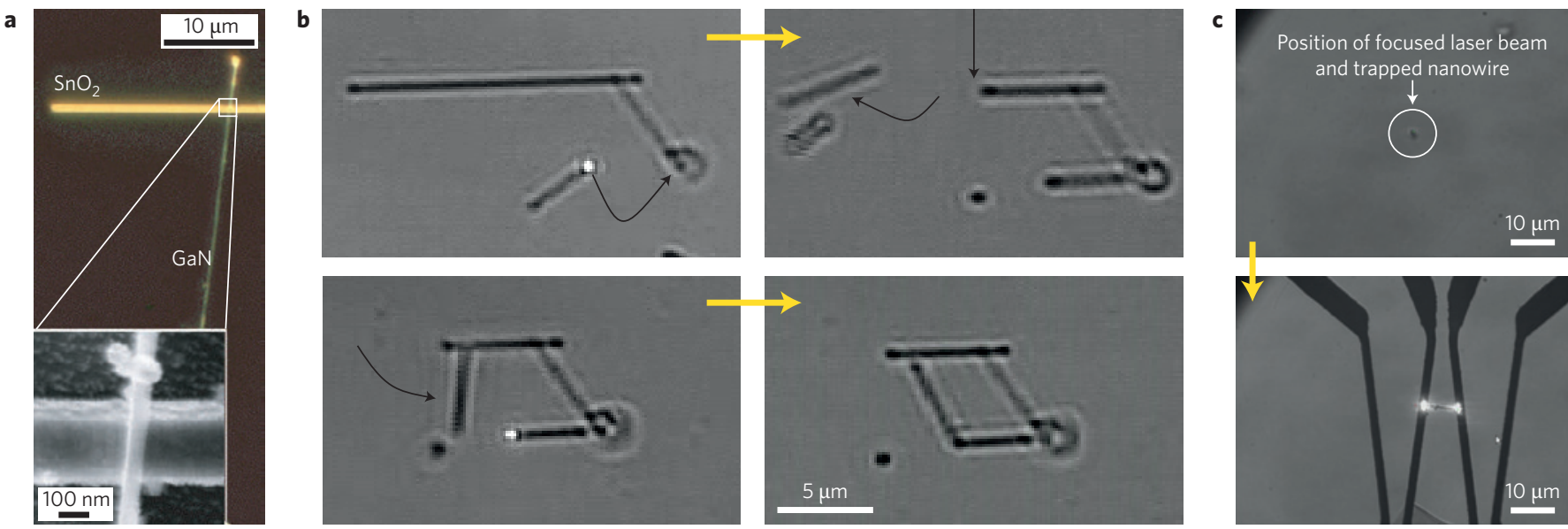

Figure 3 | Optical manipulation and placement of nanowires. a, Semiconductor nanowires can be manipulated and assembled with optical forces. The image shows a GaN nanowire laser-fused to a $\mathrm{SnO}_{2}$ nanoribbon after manipulation and deposition with optical tweezers. The inset is a scanning electron micrograph of the fused junction. $\mathbf{b}$, Assembly of a rhombus constructed from semiconductor CdS-nanowires using HOTs. This entails nanowire translation, cutting and fusion with the substrate. c, Optical tweezing of a $\ln _{2} \mathrm{O}_{3}$ nanowire (top) and placement by scanning optical tweezers, to connect two branches of a circuit (bottom). Figure reproduced with permission from: a, ref. 43, (c) 2006 NPG; b, ref. 42, (c) 2005 OSA; c, ref. 119, (c) 2009 OSA.

tweezer configuration at the trapping plane ${ }^{123-125}$. Over the past few years, HOTs have been used to manipulate and assemble nanostructures. For example, semiconducting nanowires have been translated,

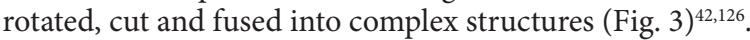

Plasmonic tweezers. In the 1990s and early 2000s, various groups theoretically suggested harnessing the enhancement associated with a plasmonic resonance to realize nanoscopic optical tweezers, for example by using the extremity of a sharp metallic tip ${ }^{127,128}$, the light transmitted through a nanohole in a metallic film ${ }^{129}$ or metallic patterns to create multiple trapping positions at the nanoscale $e^{50}$.

In 2006, Volpe et al. ${ }^{51}$ showed that surface plasmon polaritons at a glass/ $\mathrm{Au} /$ water interface produce a 40 -times increase in the optical forces on micrometre-sized dielectric particles (Fig. 4a), so that collections of such particles could self-organize in large crystals ${ }^{130}$. But a flat metal film features a homogeneous optical potential ${ }^{51}$, whereas controlled trapping of single nano-objects requires patterning of the surface to create three-dimensional confining optical potentials ${ }^{50}$. This is achieved by using properly designed metal nanostructures such as pads ${ }^{52,53}$, antennas ${ }^{54,55}$ or nanoapertures ${ }^{56,58}$. A typical optical set-up to excite plasmons is based on the Kretschmann configuration shown in Fig. 2b. With similar schemes it is also possible to arrange microscopic particles in complex configurations corresponding to the locations of metallic micropads, where a plasmonic resonance can be excited (Fig. $4 \mathrm{~d}$ ), and also to integrate such plasmonic traps with a microfluidic environment ${ }^{131}$. Other configurations based on nanoantennas allow one to localize the field intensity in hotspots ${ }^{54,55}$ (Fig. 4c). Fractal plasmonic structures ${ }^{132}$ can allow tight foci below the diffraction limit far away (hundreds of nanometres) from the metallic structures (Fig. 4d).

Plasmonic interactions can also be harnessed by using the active feedback from the interaction between the optical tweezer beam and the trapped particle. It is possible to overcome the scaling of the optical forces with the third power of the object size, as well as the increase in Brownian fluctuation, by making use of an optical trap realized with a nanoaperture in a metal film (Fig. 4e) in which the particle itself has a strong influence on the local electric field. The particle thus has an active role in the trapping mechanism, increasing the stiffness of the trap only when the particle tries to escape $e^{58}$. Plasmonic double nanoapertures were also used for optical trapping of single proteins, paving the way to direct optical manipulation of smaller objects ${ }^{56}$. Note that whenever plasmonic nanostructures are involved, the problem of heating must be faced. Wang et al..$^{57}$ have described a method of reducing heat in a plasmonic trap by using a heat sink integrated with the optical structure.

Photonic force microscopy. A photonic force microscope $(\mathrm{PFM})^{133-135}$ is a scanning probe technique based on optical tweezers (Fig. 5). This concept was originally developed when scanning a dielectric particle trapped on a surface and observing how its Brownian motion in the trap was modified by the probe-sample interaction ${ }^{133}$. In this way, it was possible to measure extremely small forces down to femtonewtons, as well as image surface features below the trapping light diffraction limit ${ }^{133,134,136,137}$.

The motion of a trapped particle subject to thermal fluctuations can be modelled in one dimension by the overdamped Langevin equation ${ }^{115}$ :

$$
\frac{\mathrm{d} x(t)}{\mathrm{d}(t)}=-\frac{K_{x}}{\gamma} x(t)+\sqrt{2 D} W(t)
$$

where $x(t)$ is the particle position, $K_{x}$ the stiffness of the optical trap, $\gamma$ the friction coefficient, $D$ the Stokes-Einstein diffusion coefficient and $W(t)$ a white noise. When dealing with quantitative force measurements, it is crucial to calibrate the optical trap stiffness, $K_{x}$. This calibration can be obtained by measuring the Brownian trajectory of the optically trapped particle using the deflection of the trapping beam onto a quadrant photodiode ${ }^{136}$, a device that allows one to map the particle trajectory ${ }^{135}$. These trajectories are typically analysed by fitting the autocorrelation function of $x(t)$ to an exponential ${ }^{138}$ : the characteristic decay relaxation time of the autocorrelation function is $\tau=\gamma / K_{x}$. Deriving the value of $\gamma$ from hydrodynamics, it is then possible to measure $K_{x}$. Alternatively, it is possible to perform this analysis in the frequency domain using the power spectral density of $x(t)$ (ref. 136), which can be fitted to a Lorentzian lineshape ${ }^{139}$. When dealing with force measurements in the presence of diffusion gradients on the probe, such as close to boundaries or objects, some correction terms are necessary ${ }^{18}$, and these are more significant for a nanometre-sized probe ${ }^{140}$.

Using an optically trapped particle as a PFM probe may be advantageous in imaging of soft structures ${ }^{135}$, because the trap stiffness is low $\left(10^{-3} \text { to } 1 \mathrm{pN} \mathrm{nm}^{-1}\right)^{15}$ compared with that of an atomic force microscope cantilever $\left(10 \text { to } 10^{5} \mathrm{pN} \mathrm{nm}^{-1}\right)^{15}$, and in volumetric imaging ${ }^{137}$ at high temporal resolution (tens of kilohertz sampling rates ${ }^{137}$ ), which can be achieved by three-dimensional particle 

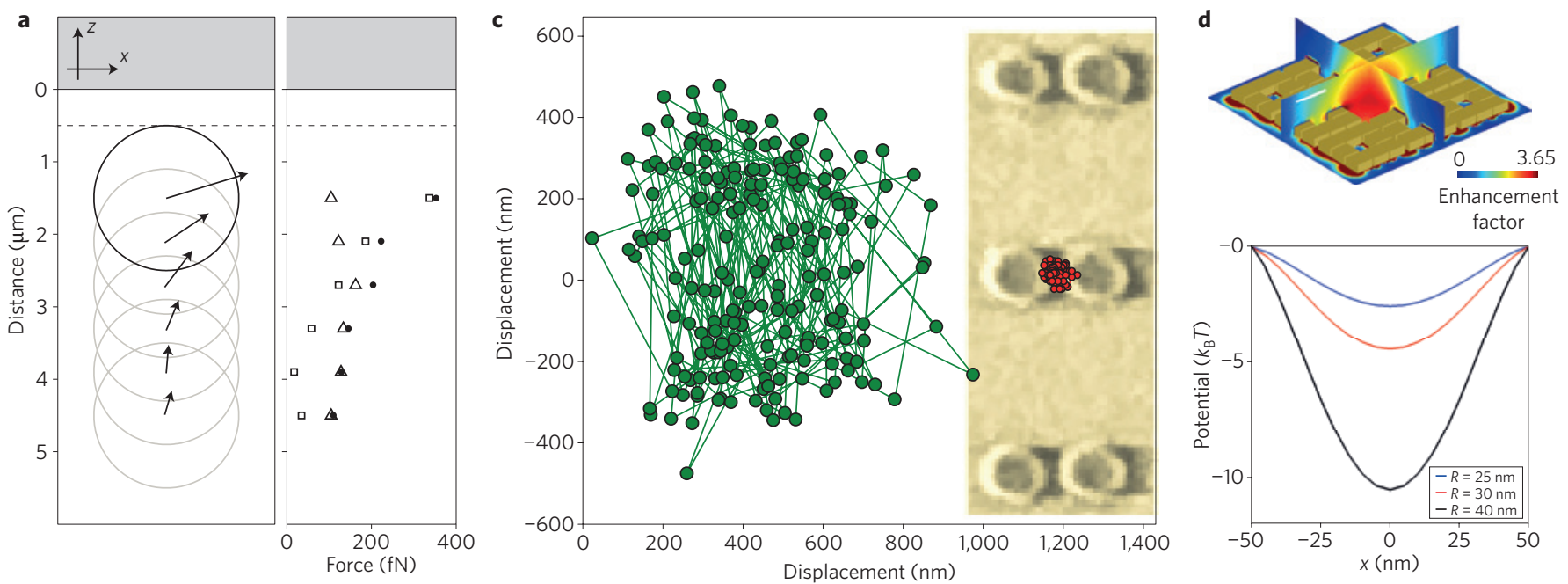

b

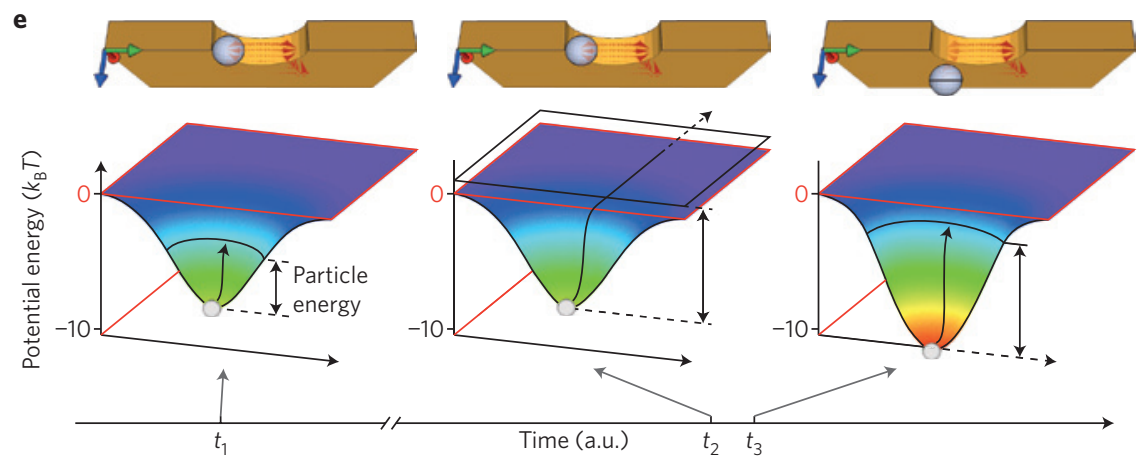

Figure 4 | Plasmonic tweezers. a, Plasmonic optical tweezers make use of the enhanced electromagnetic fields arising when metallic nanostructures are illuminated with the appropriate wavelength and polarization. The left panel shows the force vector whereas the right panel plots the module (circles), $x$ component (squares) and $z$ component (triangles) of the force as a function of distance from the surface of a $2 \mu \mathrm{m}$ bead. The surface is represented by the grey area at the top, at 0 distance. $\mathbf{b}$, Arrangement of polystyrene microparticles at a patterned Au/water interface where a plasmonic resonance is excited: the particles are selectively trapped at the Au micropads. c, The trajectory of a 200-nm nanoparticle trapped between two metallic nanodots on a planar surface (red dots) is greatly confined compared with when it is free (green dots). d, Upper panel: Subdiffraction focusing by a Sierpinsky plasmonic nanocarpet: that is, an arrangement of plasmonic nanostructures according to a fractal geometry similar to the Sierpinsky carpet ${ }^{131}$. Lower panel: Associated optical forces acting on a 30-nm dielectric polystyrene nanoparticle. e, Schematic of trapping set-up for a 310-nm aperture in a 100-nm Au film and 100-nm polystyrene spheres in water: as the particle is about to escape the nanohole, the optical potential becomes deeper and pulls it back. Figure reproduced with permission from: a, ref. 51, (c) 2006 APS; b, ref. 52, (c) 2007 NPG; c, ref. 54, (c) 2008 NPG; d, ref. 132, (c) 2011 OSA; e, ref. 168 , (c) 2011 NPG.

tracking, instead of the two-dimensional video-rate projection on a camera $^{135}$. The combination of sensitive position detection and low spring constant leads to a force resolution of few femtonewtons, far surpassing other scanning probe techniques ${ }^{15}$. Spatial resolution, however, may be limited by particle size and thermal fluctuations. The use of linear nanostructures as probes is therefore crucial to increase resolution ${ }^{62-64}$, as the combination of their nanometric transverse size and micrometric length is key to allow stable optical tweezing (even at very low laser power) while maintaining high lateral resolution ${ }^{34,59-61}$. Light-emitting or light-guiding nanostructures (Fig. 5a), such as potassium niobate nanowires ${ }^{44}$ or polymer nanofibres ${ }^{41}$, offer tunable nanoscale light sources that could permit subwavelength microscopy ${ }^{44}$.

In the case of low-dimensional structures, for example CNTs, nanowires, graphene or other two-dimensional crystals, the reduced symmetry means that the optical torque is crucial in determining their alignment and orientation with respect to the beam propagation or polarization directions ${ }^{89,91,93}$. Similarly, whereas for spherical nanostructures the detector signals are combined so that they are proportional to the centre-of-mass displacements ${ }^{20,31}$, non-spherical ones also contain angular information ${ }^{35,38,141}$. In particular, in linear nanostructures, length is the key parameter that regulates forces, torques and hydrodynamics ${ }^{46,47}$. These combined force and torque measurements allow optical tweezer calibration when using nonspherical particles as femtonewton force-sensing probes $^{35,60}$ (Fig. 5).

Force lithography and placement. The combination of laser manipulation and photopolymerization allows one to build threedimensional structures using $\mathrm{NPs}^{142}$, and to place them on a substrate $^{142,143}$. For example, ref. 142 used an infrared trapping laser beam to collect, near its focus, NPs suspended in solution, and then applied a ultraviolet laser to induce photopolymerization of a monomer, also present in solution, around the NPs. It is also possible to use optical tweezers to trap and position single NPs: for example to manipulate, assemble and fuse different semiconductor nanowires ${ }^{43}$ (Fig. 3a). Controlled deposition of optically trapped $\operatorname{In}_{2} \mathrm{O}_{3}$ nanowires was realized by fast-scanning the trapping beam to rotate the nanowires and connect two branches of a circuit ${ }^{119}$ (Fig. 3c). Optical tweezers have also been used to trap and place AuNPs on a substrate with a positioning error of $\sim 100 \mathrm{~nm}$ (ref. 143), largely owing to Brownian fluctuations. This technique can be parallelized using, for example, HOTs, as in ref. 144 where HOTs were used to deposit AuNPs on glass (Fig. 2d), and in ref. 145 where they were used to organize zeolite crystals. 

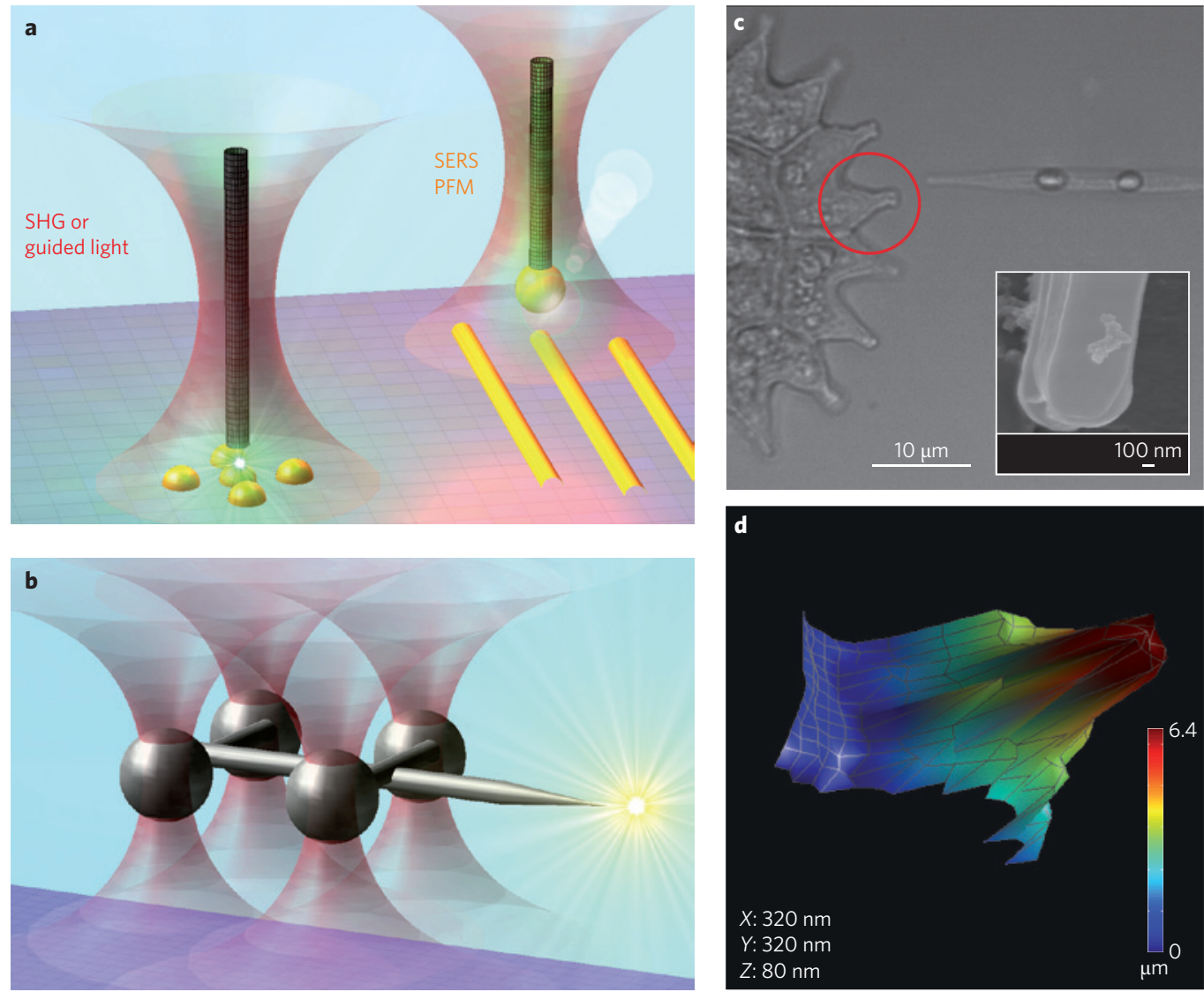

Figure 5 | Photonic force microscope. a, Schemes for the realization of a PFM with functional nanostructures. Left: Optical active probes that act as light guides (such as in polymer nanofibres ${ }^{41}$ ) or produce SHG (as in potassium niobate nanowires ${ }^{44}$ ) are trapped and scanned over a sample to deliver both morphological and spectroscopic information in a liquid environment. Right: A SERS-PFM based on hybrid (metal-dielectric) probes can combine singlemolecule detection with morphological information. $\mathbf{b}$, More complex and composite probes, made by two-photon lithography, are used with HOTs to reach nanometre resolution combined with femtonewton force sensing $60,61,64 . \mathbf{c}, \mathbf{d}$, Shaped living probes (diatom Nitzschia subacicularis) are trapped with HOTs (c) and used to image other living organisms (Pseudopediastrum; d). Panels c,d reproduced with permission from ref. 62, (c) 2011 IOP.

Another approach is optical-tweezers-assisted nanopatterning, where a micrometre-sized dielectric sphere is positioned close to a surface and acts as a near-field objective lens to focus the laser for pulsed laser processing ${ }^{146}$. With this technique, patterns with features down to $\sim 100 \mathrm{~nm}$ have been generated ${ }^{146}$. Surfaces with nonconstant height have also been patterned by taking advantage of the non-diffracting properties of Bessel beams ${ }^{147}$.

\section{Spectroscopy of nanostructures in optical traps}

Spectroscopic optical tweezers (SOTs) are obtained by integrating optical tweezers with spectroscopic functionalities. SOTs allow one to study the chemical properties of a single nanostructure by probing in situ its vibrational (Raman) ${ }^{33,36,38}$, electronic (photoluminescence) ${ }^{45,49}$, plasmonic (scattering) (6, $148,149^{1}$ or nonlinear (for example two-photon photoluminescence ${ }^{48,72}$ ) properties. In this way, it is possible to select nanostructures with specific physicochemical properties, for example SWNTs with certain chiral indices ${ }^{36}$, out of an ensemble of NPs with different properties. A typical SOT set-up incorporates two optical beams, as shown in Fig. 6a: one to trap the nanostructure and one to excite it. In some cases, it is possible to use a single beam for both tasks ${ }^{36,38}$. The single-beam configuration is, indeed, simpler and more stable, although the two-beam arrangement offers more versatility to trap, manipulate and excite specific zones of the object, by displacing the trapping and excitation beams independently ${ }^{4}$. Trapping is often accomplished with a NIR laser to minimize photodamage of biomaterials ${ }^{150}$, whereas visible light is often used for excitation of the nanostructures ${ }^{45,72}$.
Photoluminescence tweezers. Photoluminescence spectroscopy is an optical method to probe the electronic and structural properties of nanomaterials, and has been successfully integrated into optical tweezers. For example, ref. 45 investigated the structural properties of single InP-nanowires in liquid by combining 1,064-nm optical tweezers with 514.5-nm photoluminescence excitation. Based on the energy maximum in the photoluminescence emission, it was possible to use the spectra of individually trapped nanowires to differentiate nanowires with different structure (zincblende, wurtzite and mixed phases $)^{45}$. Moreover, by implementing a HOT with a SLM, Wang et al. ${ }^{49}$ scanned the excitation spot along the trapped nanowires, mapping structural inhomogeneities and allowing sorting of specific nanowires before their incorporation into devices. Two-beam SOTs were also used to investigate the nonlinear photoexcitation in optically trapped InP-nanowires ${ }^{72}$. Under strong $\left(\sim 100 \mathrm{MW} \mathrm{cm}^{-2}\right)$ excitation at $1,064 \mathrm{~nm}$, second-harmonic generation at $532 \mathrm{~nm}$ was observed from individual nanowires, together with band-edge photoluminescence emission at $890 \mathrm{~nm}$ due to two- and three-photon absorption $^{72}$. From the redshift between the two-photon absorption photoluminescence and the direct absorption photoluminescence (excited at $514.5 \mathrm{~nm}$ ), it was possible to probe band-filling at the single nanowire level ${ }^{72}$. Optical manipulation of semiconductor nanowires with such techniques offers an attractive route for the development of devices with engineered electronic properties, and for component-wise assembly of nanophotonic devices ${ }^{151}$.

Perovskite alkaline niobate nanowires have attracted much attention for their interesting nonlinear optical response ${ }^{44,48}$ and their use 
as mechano-optical probes ${ }^{44}$. The polarization-dependent second harmonic generation (SHG) from optically trapped nanowires has been studied with SOTs. The nanowires showed waveguiding that enabled the SHG signal to propagate at the nanowire apex, thus acting as a nanoscale light source for $\mathrm{PFM}^{44}$ (Fig. 5a).

Photoluminescence spectroscopy is one of the most important tools for characterization of SWNTs ${ }^{152}$. Photoluminescence spectra allow the determination of the chiral indices ${ }^{152}$, as well as providing information on the bundling through the study of exciton energy transfer ${ }^{153}$ and the interaction with the local environment (dielectric screening shifts the photoluminescence ${ }^{154}$ ). Spectroscopic optical tweezers allow one to perform single bundle analysis in solution. Figure $5 \mathrm{~b}$ shows the photoluminescence of a single bundle dispersed in a water/taurodeoxycholate solution ${ }^{114}$, optically trapped and probed in a single beam SOT $(\lambda=633 \mathrm{~nm})$, enabling chirality assignment.

Raman tweezers. Raman tweezers are realized by coupling a Raman spectrometer with optical tweezers, thus allowing the chemical and physical analysis of a trapped particle through its vibrational fingerprints. They were first introduced for the investigation of biological material ${ }^{150}$ and were shown to discriminate between living and dead yeast cells ${ }^{150}$. The ability of Raman tweezers to trap and analyse individual nanostructures was further demonstrated on 40-nm polystyrene beads ${ }^{66}$.

The potential of Raman tweezers as a tool for analysis and manipulation of nanostructures in liquid has been demonstrated on carbon nanostructures ${ }^{33}$, and they have been used to selectively trap and aggregate SWNTs with specific chiralities ${ }^{36}$. This was done by focusing a 633-nm beam on a solution containing dispersed tubes, and mapping the increase as a function of time of radial breathing modes related to SWNTs of specific chiralities ${ }^{36}$.

A very desirable step in graphene technology is the development of techniques capable of manipulating individual flakes in solution, sorting them as a function of shape and number of layers, and accurately positioning them to design devices with controlled properties. Raman tweezers are well suited for this, as Raman spectroscopy allows one to extract structural and electronic information on individual flakes ${ }^{155,156}$, as first implemented in ref. 38 (Fig. 6c).

Rayleigh spectroscopy and SERS. Rayleigh spectroscopy measures the spectral dependence of the elastic light scattering cross-section and probes plasmon resonances in $\mathrm{MNPs}^{95}$. Metallic nanoparticles are interesting as optically resonant nanoantennas, capable of spatially confining and enhancing the local electromagnetic field by orders of magnitude ${ }^{95}$. Nanoparticle dimers, trimers and fractal aggregates with new functionalities and higher field enhancement capabilities ${ }^{157}$ can, in principle, be created using optical forces. By combining Rayleigh scattering with optical tweezers, Prodan et al. ${ }^{158}$ showed plasmon hybridization, caused by the close encounter between a trapped AgNP and an immobilized one ${ }^{148}$. The plasmon resonance energy shift ${ }^{157}$ can be used as a parameter to quantitatively study the interaction potential between colloidal NPs in optical tweezers ${ }^{28}$ and reconstruct the interparticle potential energy landscape as a function of distance, allowing one to tune the optical interaction between the NPs in the dimer. Optical forces were also shown to be strongly affected by near-field coupling among NPs simultaneously trapped ${ }^{149}$. The coupling was found to strengthen the NP interaction with the trapping light, causing a gradual shift of the plasmon resonance towards the laser wavelength ${ }^{149}$. This resulted in a thermal destabilization of the system because of the enhanced light absorption and consequent overheating of the water layer around the NPs ${ }^{149}$.

Surface-enhanced Raman spectroscopy (SERS) takes advantage of the local field enhancement offered by optically resonant MNPs to amplify the Raman signal ${ }^{95}$ and allows in principle for high-sensitivity label-free identification of molecular species ${ }^{159}$. Optically coupled MNPs are among the most efficient substrates for SERS of molecular adsorbates ${ }^{157}$. Optical tweezers have proven to be an effective tool to create SERS-active metal nanocolloid aggregates ${ }^{68}$. Spectroscopic optical tweezers therefore have great potential for ultrasensitive, label-free, molecular recognition in liquids ${ }^{67}$. Optical forces have been used ${ }^{68}$ to bring two AgNPs into near-field contact in a liquid solution containing thiophenol $(10 \mu \mathrm{M})$, creating a SERS-active dimer capable of strongly enhancing the Raman signal relative to the case of a single trapped AgNP. Repulsive optical forces have also been used to aggregate AgNPs on glass coated with 3-aminopropyltrimethoxysilane, so to form SERS-active aggregates. This has been used ${ }^{67}$ to detect Rhodamine $6 \mathrm{G}$ in solution down to $0.1-\mu \mathrm{M}$ concentration.

Biomolecules, such as proteins or nucleic acids, find in liquid their natural, functional environment. The rapid, ultrasensitive, label-free detection of pathology biomarkers in body fluids is a field in which plasmonic nanosensors can find several applications ${ }^{160}$. Two different concepts of SERS-based nanosensors for the detection of biomolecules in liquid have recently been demonstrated using $\mathrm{SOTs}^{70,71}$. In the first, a double-stranded deoxyribonucleic acid (DNA) molecule, tagged with biotin and dioxydenine at each end, was anchored between two optically trapped $\left(\lambda_{\text {trap }}=1,064 \mathrm{~nm}\right)$ polystyrene beads coated with streptavidin and anti-dioxydenine ${ }^{70}$. The DNA was thus suspended in a solution containing SERS-active Ag nanocolloids and excited with a second laser beam $\left(\lambda_{\text {exc }}=785 \mathrm{~nm}\right)$ that allowed recovery of the enhanced signal of three vibrational bands of DNA. In the second concept (Fig. 6d), Au nanocolloidal aggregates optically trapped in a single-beam SOT $(785 \mathrm{~nm})$ were used to detect proteins adsorbed on their surface ${ }^{71}$.

A PFM can benefit from the ability to fabricate SERS-active nanoprobes, paving the way for local enhanced spectroscopy of biological surfaces. A route to accomplish this is to design special SERS-active probes consisting of metal colloids (Ag and $\mathrm{Au}$ ) tightly bound to micrometric silica beads ${ }^{69}$ or to nanowires (see, for example, Fig. 5a), with reduced thermal fluctuations compared with individual MNPs ${ }^{46}$. By optically manipulating and exciting such silica hybrid probes in close contact with the surface of cells incubated in emodin (concentration $2 \mu \mathrm{M}$ ) within a single beam optical tweezers $\left(\lambda_{\text {laser }}=785 \mathrm{~nm}\right)$, it is possible to detect the SERS fingerprint of membrane emodin molecules ${ }^{69}$.

SERS-optical tweezers couple high molecular sensitivity with the contact-less, label-free, three-dimensional capability of operation in liquids. SERS-active probes can be highly specific because functionalized probes allow selective interaction with specific sample sites. Thus, they represent a promising tool for the development of next-generation biosensors capable of detecting biomolecules and investigating biological samples in their natural environment. The combination of optical tweezers and optical injection of NPs inside living cells has recently been demonstrated ${ }^{161}$. Photoporation ${ }^{162}$ - that is, the process of creating a transient pore on a cell membrane with a focused laser beam - was used for the targeted delivery of 100-nm AuNPs into a specific region of the interior of an individual mammalian cell ${ }^{161}$. This provides a new all-optical methodology for internalizing nanobiosensors within specific intracellular regions.

\section{Optomechanics with levitated nanostructures}

Optomechanics is the study of mechanical motion induced by optical forces ${ }^{163}$. Recently, much effort has been devoted to the study of quantum phenomena at mesoscopic or macroscopic length scales, and to the development of techniques bridging the gap between laser cooling of atomic species and optical trapping of colloidal materials ${ }^{74}$. The aim is to uncover and exploit quantum effects, 
a

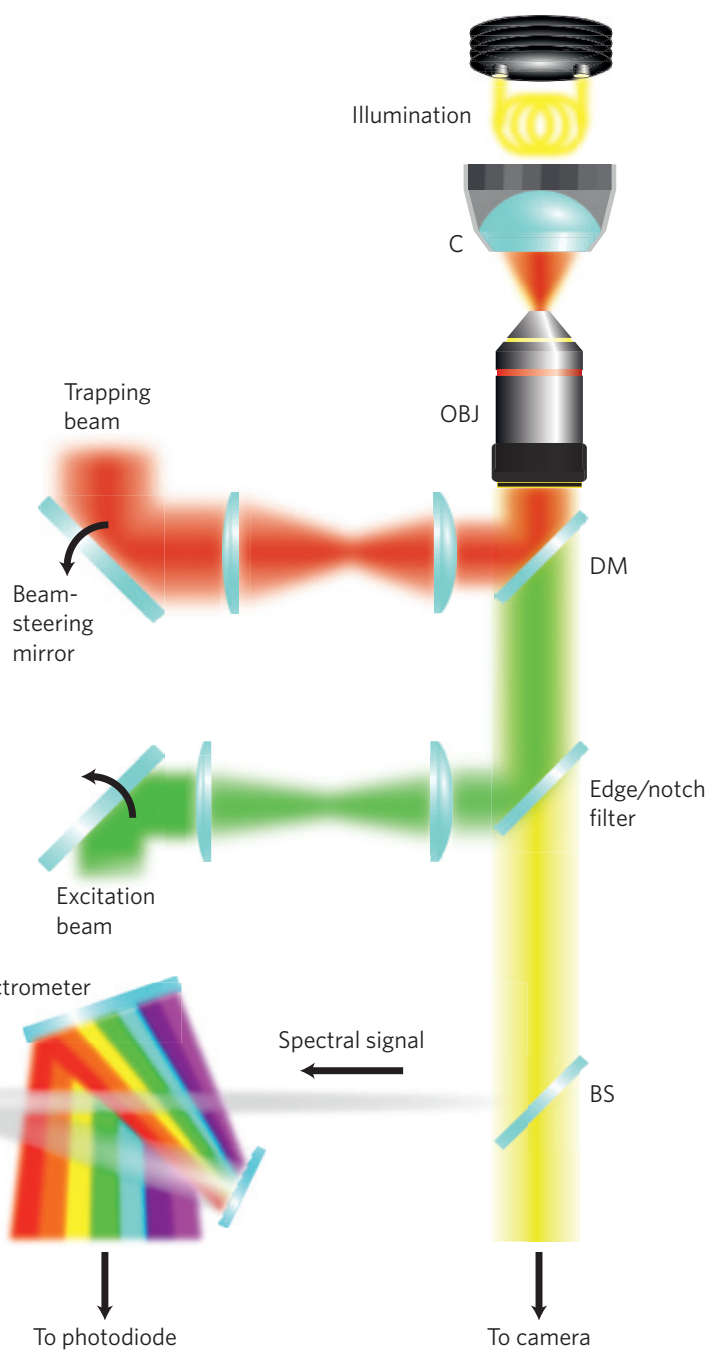

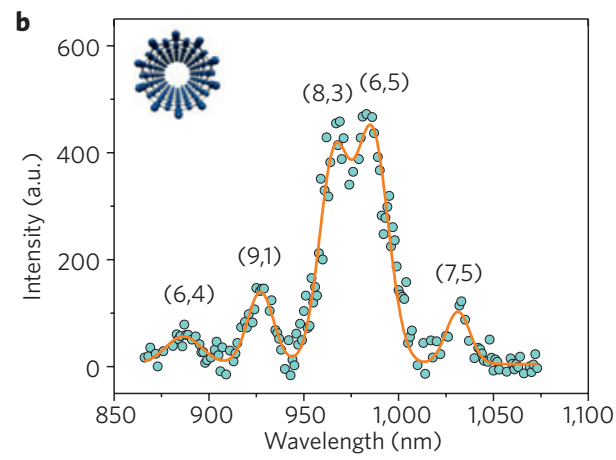

c

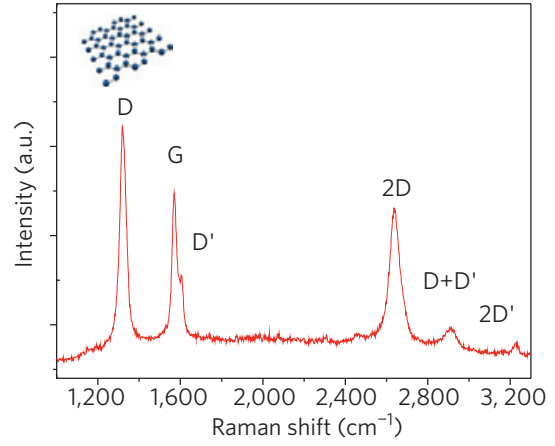

d

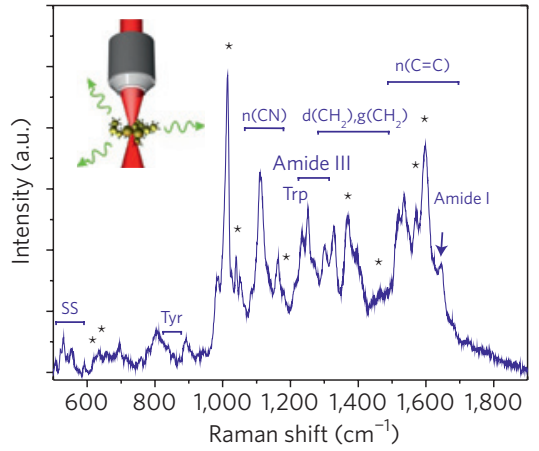

Figure 6 | Spectroscopy of nanostructures in optical traps. a, Set-up integrating optical tweezers and spectroscopy. The trapping and excitation beams are focused through a high-numerical-aperture objective lens (OBJ). The trapping and excitation beams are combined using dichroic mirrors (DM). For scattering spectroscopy the sample can also be excited using a halogen lamp focused by a dark-field condenser (C) from the top (IIlumination). The signal is collected through the trapping objective lens. Notch/edge filters are used to cut out the elastic scattering at the excitation/trapping wavelengths. A beam splitter (BS) divides the imaging light from the spectral signal. Grating spectrometers provided with CCD cameras or avalanche photodiodes acquire the spectroscopy signal. b. Example of a photoluminescence spectrum of a SWNT bundle confined by optical tweezers (data from ref. 34). c, Raman spectrum of an optically trapped graphene flake with 633-nm trapping and excitation wavelength. d, SERS of bovine serum albumin (BSA) proteins performed in liquid by optically trapping Au colloidal aggregates on which the protein is adsorbed. The enhanced BSA peaks are indicated in blue(SS, disulfide bridges; Ty, tyrosine; Trp, tryptophan, amide bands, stretching modes); the asterisks indicate the SERS signal from pyridine. Figures adapted with permission from: c, ref. 38, (c) 2010 ACS; d, ref. 71, (c) 2011 ACS.

such as entanglement ${ }^{74}$, quantum superposition of motional states ${ }^{73}$, and long quantum coherence ${ }^{74}$ in systems larger than atoms or molecules. One technique suggested for reaching the quantum regime is NP optical levitation in a high-finesse cavity ${ }^{73}$ (Fig. 7a). In this scheme, a subwavelength particle is held by optical tweezers inside the cavity. A second laser excites a cavity mode that couples with the trapped particle's centre-of-mass motion. This optomechanical coupling shifts the cavity modes yielding a velocity-dependent force responsible for laser cooling ${ }^{74,75}$.

Experimentally, the first step towards this goal is the trapping and laser cooling of nanostructures in vacuum, extending the methodologies used for neutral atoms and ions ${ }^{11}$ (Fig. 7b,c,d). Kane ${ }^{164}$ has reported levitation of graphene flakes. Starting from a liquid suspension of graphene $e^{38,165}$, charged flakes were injected into an ion trap ${ }^{10,11}$ using electrospray ionization ${ }^{164}$. By monitoring the light scattering from the flakes (Fig. $7 \mathrm{~d}$ ) it was possible to infer the particle dynamics in the trap, the starting point for implementing laser cooling ${ }^{166}$.

Gieseler et $a .^{76}$ demonstrated laser cooling of a silica NP (Fig. 7b). In this case, optical tweezers were operated in vacuum, where motion of the particle in the trap is underdamped ${ }^{166}$. Light scattered by the particle was monitored with photodiodes to infer the particle motion, then used in a feedback loop that modified the trapping light intensity ${ }^{76}$. This was adjusted so that the trap stiffness increased when the particle moved away from its equilibrium, and reduced otherwise. The effective temperature (as low as $\sim 50 \mathrm{mK}$ ) was then measured by observing residual thermal fluctuations ${ }^{166}$. In this feedback cooling scheme ${ }^{166}$ the particle internal structure has no role. But just as the internal structure of atoms enables sub-Doppler cooling mechanisms ${ }^{11}$, the engineering of complex coupled or hybridized nanostructures enables the interaction with light to be modified so that even lower temperatures $(\mu \mathrm{K})$ can be achieved ${ }^{167}$. 
a
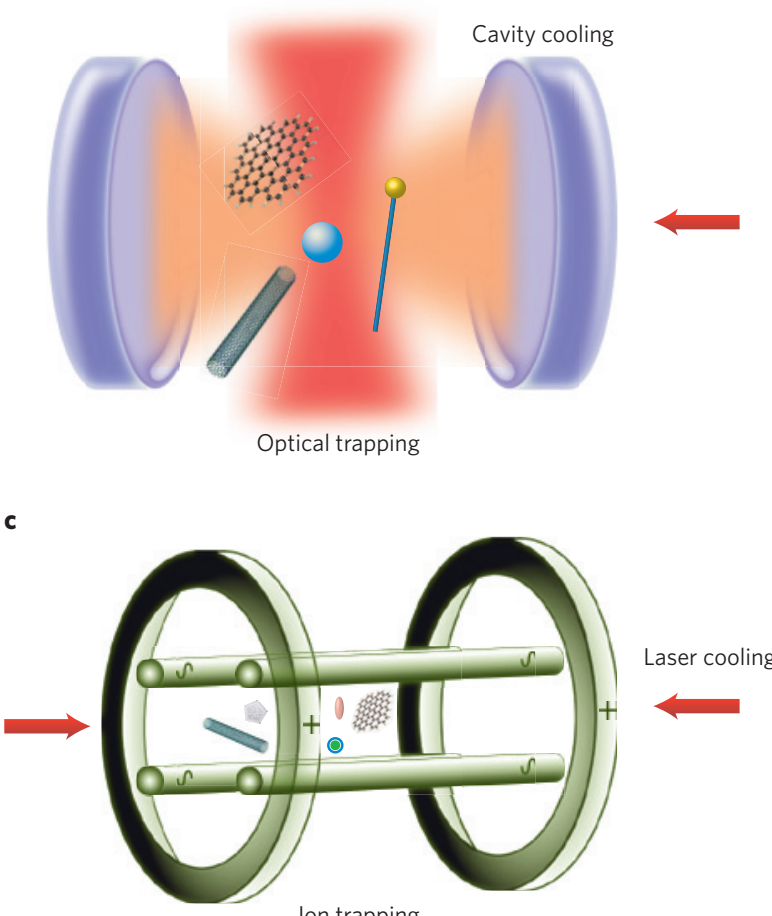

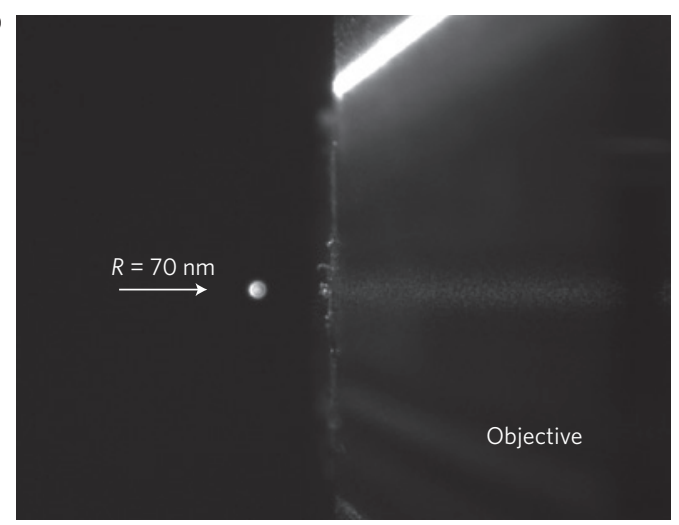

d

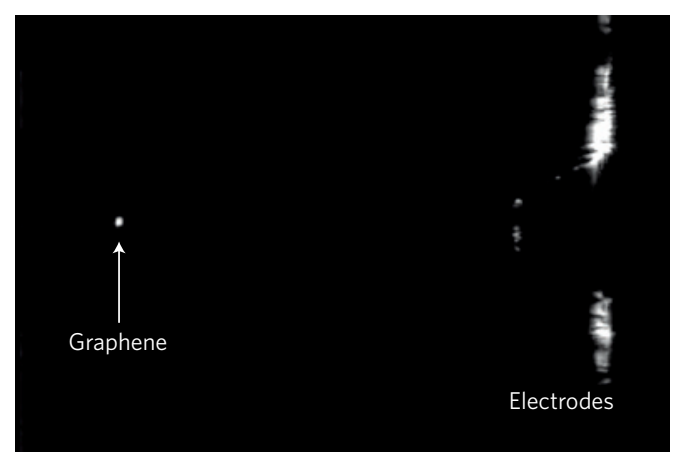

Figure 7 | Nanoparticle levitation and laser cooling. a, Optomechanics with NPs trapped and cooled in a high-finesse optical cavity. Nanostructures are trapped by optical tweezers (red). Their centre-of-mass motion is confined by a harmonic potential with characteristic frequency $\omega_{\text {trap }}$ The interaction with

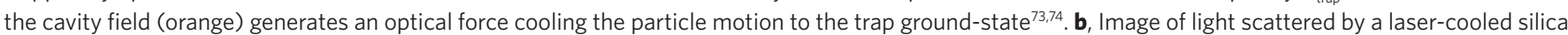
NP confined in optical tweezers. This light is detected to reconstruct the particle motion in the trap. A feedback cooling scheme, where the same beam is used for trapping and cooling, is then used to reach temperatures as low as $50 \mathrm{mK}$ for the particle centre-of-mass motion. $\mathbf{c}$, Scheme for ion trapping and laser cooling of nanostructures. Particles can be injected in vacuum by electrospraying ${ }^{164}$ a liquid suspension (such as that used to disperse graphene ${ }^{38,165}$ or $\mathrm{CNTs}^{35,114}$ ), and confined using electrostatic trapping as for ions ${ }^{11,10}$. Radiation pressure can then laser-cool the centre-of-mass motion ${ }^{166}$, so as to reach the quantum ground-state of the trap. d, Image of light scattered by a graphene flake in an electric quadrupole ion trap. Graphene flakes were also put into rotation by shining circularly polarized light in the trap region. Figure reproduced with permission from: b, ref. 76 , (c) 2012 APS; d, ref. 164 , (c) 2010 APS.

\section{Perspective}

In the context of the ongoing trend towards miniaturization of technology towards the nanoscale, optical trapping and manipulation of nanostructures can open new and exciting possibilities for assembly, characterization and optical control of nanodevices and biomolecules. As discussed above, there have already been considerable advances in this direction, for example with the development of techniques to perform spectroscopy on single molecules ${ }^{69-71}$, and to probe forces with femtonewton sensitivity $34,60,63,64$. Another goal within reach is coherent manipulation of a single levitated nanostructure, or entanglement on multiple nanoparticle systems, to gain a new perspective on the quantum regime applied to mesoscopic objects at room temperature. Optomechanical cooling schemes have already made progress towards the demonstration of quantized nanoparticle motional states ${ }^{76}$.

The realization of these goals will require the development of new techniques to manipulate nanoparticles beyond those currently available. Several barriers will need to be overcome: nanoparticles will need to be manipulated with subnanometre accuracy in order, for example, to develop new integrated devices. But reducing the trapping volume to the nanoscale is just part of the challenge. The ability to probe and control what happens in the trap is still missing. Effects associated with heating of plasmonic structures must be mitigated by integration of cooling schemes. For optimal control and regulation of biomolecular interactions, specificity in single-molecule trapping is required. It will be necessary to manipulate and assemble large numbers of particles to reach high-throughput and cost-efficient production. This could be achieved by self-assembly of elementary building blocks. The development of autonomous nanodevices capable of their own locomotion and of exploring their environment can be envisaged. In this context, optical manipulation of individual nanoparticles will play a crucial role in the development and characterization phase, but more powerful, and largely new, parallel optical manipulation techniques will also be essential. Ideally, these new trapping schemes for nanostructures should be as flexible and widely applicable as optical tweezers have proven to be for micrometre-scale material.

Received 6 June 2013; accepted 12 September 2013; published online 7 November 2013

\section{References}

1. Poynting, J. H. On the transfer of energy in the electromagnetic field. Phil. Trans. R. Soc. Lond. 175, 343-361 (1884).

2. Lebedev, P. Untersuchungen über die druckkräfte des lichtes. Ann. Phys. 311, 433-458 (1901).

3. Nichols, E. F. \& Hull, G. F. A preliminary communication on the pressure of heat and light radiation. Phys. Rev. 13, 307-320 (1901).

4. Ashkin, A. History of optical trapping and manipulation of small neutral particle, atoms, and molecules. IEEE J. Selected Topics Quant. Electr., 6, 841-856 (2000).

5. Ashkin, A. Acceleration and trapping of particles by radiation pressure. Phys. Rev. Lett. 24, 156-159 (1970).

6. Ashkin, A. Atomic-beam deflection by resonance-radiation pressure. Phys. Rev. Lett. 25, 1321-1324 (1970).

7. Chu, S. The manipulation of neutral particles. Rev. Mod. Phys. 70, 685-706 (1998).

8. Cohen-Tannoudji, C. Manipulating atoms with photons. Rev. Mod. Phys. 70, 707-719 (1998). 
9. Phillips, W. D. Laser cooling and trapping of neutral atoms. Rev. Mod. Phys. 70, 721-740 (1998).

10. Leibfried, D., Blatt, R., Monroe, C. \& Wineland, D. Quantum dynamics of single trapped ions. Rev. Mod. Phys. 75, 281-324 (2003).

11. Foot, C. J. Atomic Physics (Oxford Univ. Press, 2005).

12. Ashkin, A., Dziedzic, J., Bjorkholm, J. \& Chu, S. Observation of a single-beam gradient optical trap for dielectric particles. Opt. Lett. 11, 288-290 (1986).

13. Dholakia, K. \& Čižmár, T. Shaping the future of manipulation. Nature Photon. 5, 335-342 (2011)

14. Padgett, M. \& Bowman, R. Tweezers with a twist. Nature Photon. 5, 343-348 (2011).

15. Neuman, K. C. \& Nagy, A. Single-molecule force spectroscopy: optical tweezers, magnetic tweezers and atomic force microscopy. Nature Methods 5, 491-505 (2008).

16. Gordon, J. P. Radiation forces and momenta in dielectric media. Phys. Rev. A 8, 14-21 (1973).

17. Purcell, E. M. \& Pennypacker, C. R. Scattering and absorption of light by nonspherical dielectric grains. Astrophys. J. 186, 705-714 (1973).

18. Volpe, G., Helden, L., Brettschneider, T., Wehr, J. \& Bechinger, C. Influence of noise on force measurements. Phys. Rev. Lett. 104, 170602 (2010).

19. Svoboda, K. \& Block, S. M. Optical trapping of metallic Rayleigh particles. Opt. Lett. 19, 930-932 (1994).

20. Hansen, P. M., Bhatia, V. K. L., Harrit, N. \& Oddershede, L. Expanding the optical trapping range of gold nanoparticles. Nano Lett. 5, 1937-1942 (2005)

21. Bosanac, L., Aabo, T., Bendix, P. M. \& Oddershede, L. B. Efficient optical trapping and visualization of silver nanoparticles. Nano Lett. 8, 1486-1491 (2008).

22. Pelton, M. et al. Optical trapping and alignment of single gold nanorods by using plasmon resonances. Opt. Lett. 31, 2075-2077 (2006).

23. Toussaint, K. C. et al. Plasmon resonance-based optical trapping of single and multiple Au nanoparticles. Opt. Express 15, 12017-12029 (2007).

24. Selhuber-Unkel, C., Zins, I., Schubert, O., Sonnichsen, C. \& Oddershede, L. B. Quantitative optical trapping of single gold nanorods. Nano Lett. 8, 2998-3003 (2008).

25. Dienerowitz, M., Mazilu, M., Reece, P., Krauss, T. \& Dholakia, K. Optical vortex trap for resonant confinement of metal nanoparticles. Opt. Express 16, 4991-4999 (2008).

26. Jones, P. H. et al. Rotation detection in light-driven nanorotors. ACS Nano 3, 3077-3084 (2009).

27. Tong, L., Miljkovic, V. D. \& Käll, M. Alignment, rotation, and spinning of single plasmonic nanoparticles and nanowires using polarization dependent optical forces. Nano Lett. 10, 268-273 (2010).

28. Tong, L. et al. Plasmon hybridization reveals the interaction between individual colloidal gold nanoparticles confined in an optical potential well. Nano Lett. 11, 4505-4508 (2011).

29. Messina, E. et al. Plasmon-enhanced optical trapping of gold nanoaggregates with selected optical properties. ACS Nano 5, 905-913 (2011).

30. Ploschner, M., Cizmar, T., Mazilu, M., Di Falco, A. \& Dholakia, K. Bidirectional optical sorting of gold nanoparticles. Nano Lett. 12, 1923-1927 (2012).

31. Jauffred, L., Richardson, A. C. \& Oddershede, L. B. Three-dimensional optical control of individual quantum dots. Nano Lett. 8, 3376-3380 (2008).

32. Chen, Y. F. et al. Controlled photonic manipulation of proteins and other nanomaterials. Nano Lett. 12, 1633-1637 (2012).

33. Tan, S., Lopez, H. A., Cai, C. W. \& Zhang, Y. Optical trapping of single-walled carbon nanotubes. Nano Lett. 4, 1415-1419 (2004).

34. Maragò, O. M. et al. Optical trapping of carbon nanotubes. Physica E 40, 2347-2351 (2008).

35. Maragò, O. M. et al. Femtonewton force sensing with optically trapped nanotubes. Nano Lett. 8, 3211-3216 (2008).

36. Rodgers, T. et al. Selective aggregation of single-walled carbon nanotubes using the large optical field gradient of a focused laser beam. Phys. Rev. Lett. 101, 127402 (2008)

37. Pauzauskie, P. J., Jamshidi, A., Valley, J. K., Satcher, J. H. \& Wu, M. C. Parallel trapping of multiwalled carbon nanotubes with optoelectronic tweezers. Appl. Phys. Lett. 95, 113104 (2009).

38. Maragò, O. M. et al. Brownian motion of graphene. ACS Nano 4,7515-7523 (2010).

39. Twombly, C. W., Evans, J. S. \& Smalyukh, I. I. Optical manipulation of self-aligned graphene flakes in liquid crystals. Opt. Express 21, 1324-1334 (2013).

40. Geiselmann, M. et al. Three-dimensional optical manipulation of a single electron spin. Nature Nanotech. 8, 175-179 (2013).

41. Neves, A. A. R. et al. Rotational dynamics of optically trapped nanofibers. Opt. Express 18, 822-830 (2010).

42. Agarwal, R. et al. Manipulation and assembly of nanowires with holographic optical traps. Opt. Express 13, 8906-8912 (2005).
43. Pauzauskie, P. J. et al. Optical trapping and integration of semiconductor nanowire assemblies in water. Nature Mater. 5, 97-101 (2006).

44. Nakayama, Y. et al. Tunable nanowire nonlinear optical probe. Nature 447, 1098-1101 (2007).

45. Reece, P. J. et al. Combined optical trapping and microphotoluminescence of single InP nanowires. Appl. Phys. Lett. 95, 101109 (2009).

46. Irrera, A. et al. Size-scaling in optical trapping of silicon nanowires. Nano Lett. 11, 4879-4884 (2011).

47. Reece, P. J. et al. Characterisation of semiconductor nanowires based on optical tweezers. Nano Lett. 11, 2375-2381 (2011).

48. Dutto, F. et al. Nonlinear optical response in single alkaline niobate nanowires. Nano Lett. 11, 2517-2521 (2011).

49. Wang, F. et al. Resolving stable axial trapping points of nanowires in an optical tweezers using photoluminescence mapping. Nano Lett. 13, 1185-1191 (2013).

50. Quidant, R., Petrov, D. \& Badenes, G. Radiation forces on a Rayleigh dielectric sphere in a patterned optical near field. Opt. Lett. 30, 1009-1011 (2005).

51. Volpe, G., Quidant, R., Badenes, G. \& Petrov, D. Surface plasmon radiation forces. Phys. Rev. Lett. 96, 238101 (2006).

52. Righini, M., Zelenina, A. S., Girard, C. \& Quidant, R. Parallel and selective trapping in a patterned plasmonic landscape. Nature Phys. 3, 477-480 (2007).

53. Righini, M., Volpe, G., Girard, C., Petrov, D. \& Quidant, R. Surface plasmon optical tweezers: tunable optical manipulation in the femtonewton range. Phys. Rev. Lett. 100, 183604 (2008).

54. Grigorenko, A. N., Roberts, N. W., Dickinson, M. R. \& Zhang, Y. Nanometric optical tweezers based on nanostructured substrates. Nature Photon. 2, 365-370 (2008)

55. Righini, M. et al. Nano-optical trapping of Rayleigh particles and Escherichia coli bacteria with resonant optical antennas. Nano Lett. 9, 3387-3391 (2009).

56. Pang, Y. \& Gordon, R. Optical trapping of a single protein. Nano Lett. 12, 402-406 (2011).

57. Wang, K., Schonbrun, E., Steinvurzel, P. \& Crozier, K. B. Trapping and rotating nanoparticles using a plasmonic nano-tweezer with an integrated heat sink. Nature Commun. 2, 469 (2011)

58. Juan, M. L., Gordon, R., Pang, Y., Eftekhari, F. \& Quidant, R. Self-induced back-action optical trapping of dielectric nanoparticles. Nature Phys. 5, 915-919 (2009).

59. Ikin, L., Carberry, D. M., Gibson, G. M., Padgett, M. J. \& Miles, M. J. Assembly and force measurement with SPM-like probes in holographic optical tweezers. New J. Phys. 11, 023012 (2009).

60. Pollard, M. R. et al. Optically trapped probes with nanometerscale tips for femto-newton force measurement. New J. Phys 12, 113056 (2010).

61. Phillips, D. B. et al. Force sensing with a shaped dielectric microtool. Europhys. Lett. 99, 58004 (2012).

62. Phillips, D. B. et al. Surface imaging using holographic optical tweezers. Nanotechnology 22, 285503 (2011).

63. Olof, S. N. et al. Measuring nanoscale forces with living probes. Nano Lett. 12, 6018-6023 (2012)

64. Phillips, D. B. et al. An optically actuated surface scanning probe. Opt. Express 20, 29679 (2012).

65. Petrov, D. V. Raman spectroscopy of optically trapped particles. J. Opt. A: Pure Appl. Opt. 9, S139-S156 (2007).

66. Ajito, K. \& Torimitsu, K. Single nanoparticle trapping using a Raman tweezers microscope. Appl. Spectrosc. 56, 541-544 (2002).

67. Bjerneld, E. J. et al. Laser-induced growth and deposition of noblemetal nanoparticles for surface-enhanced Raman scattering. Nano Lett. 3, 593-596 (2003)

68. Svedberg, F. et al. Creating hot nanoparticle pairs for surface-enhanced Raman spectroscopy through optical manipulation. Nano Lett. 6, 2639-2641 (2006).

69. Balint, S. et al. Simple route for preparing optically trappable probes for surface-enhanced Raman scattering. J. Phys. Chem. C 113, 17724-17729 (2009).

70. Rao, S. et al. Single DNA molecule detection in an optical trap using surfaceenhanced Raman scattering. Appl. Phys. Lett. 96, 213701 (2010).

71. Messina, E. et al. Manipulation and Raman spectroscopy with optically trapped metal nanoparticles obtained by pulsed laser ablation in liquids. J. Phys. Chem. C 115, 5115-5122 (2011).

72. Wang, F. et al. Nonlinear optical processes in optically trapped InP nanowires. Nano Lett. 11, 4149-4153 (2011).

73. Romero-Isart, O., Juan, M. L., Quidant, R. \& Cirac, J. I. Toward quantum superposition of living organisms. New J. Phys. 12, 033015 (2010).

74. Chang, D. E. et al. Cavity opto-mechanics using an optically levitated nanosphere. Proc. Natl Acad. Sci. USA 107, 1005-1010 (2010).

75. Barker, P. F. \& Shneider, M. N. Cavity cooling of an optically trapped nanoparticle. Phys. Rev. A 81, 023826 (2010).

76. Gieseler, J., Deutsch, B., Quidant, R. \& Novotny, L. Subkelvin parametric feedback cooling of a laser-trapped nanoparticle. Phys. Rev. Lett. 109, 103603 (2012). 
77. Draine, B. T. The discrete-dipole approximation and its application to interstellar graphite grains. Astrophys. J. 333, 848-872 (1988).

78. Albaladejo, S., Marqués, M. I., Laroche, M. \& Sáenz, J. J. Scattering forces from the curl of the spin angular momentum of a light field. Phys. Rev. Lett. 102, 113602 (2009).

79. Borghese, F., Denti, P. \& Saija, R. Scattering from Model Nonspherical Particles (Springer, 2007)

80. Pfeifer, R. N. C., Nieminen, T. A., Heckenberg, N. R. \& RubinszteinDunlop, H. Momentum of an electromagnetic wave in dielectric media. Rev. Mod. Phys. 79, 1197-1216 (2007).

81. Mishchenko, M. I. Radiation force caused by scattering, absorption, and emission of light by nonspherical particles. J. Quant. Spectrosc. Radiat. Transfer 70, 811-816 (2001).

82. Nieminen, T. A., Rubinsztein-Dunlop, H. \& Heckenberg, N. R. Calculation and optical measurement of laser trapping forces on non-spherical particles. J. Quant. Spectrosc. Radiat. Transfer 70, 627-637 (2001).

83. Saija, R., Iatí, M. A., Giusto, A., Denti, P. \& Borghese, F. Transverse components of the radiation force on non-spherical particles in the T-matrix formalism. J. Quant. Spectrosc. Radiat. Transfer 94, 163-179 (2005).

84. Borghese, F., Denti, P., Saija, R. \& Iatì, M. A. Optical trapping of non-spherical particles in the T-matrix formalism. Opt. Express 15, 11984-11998 (2007)

85. Borghese, F., Denti, P., Saija, R. \& Iatì, M. A. Radiation torque on non-spherical particles in the transition matrix formalism. Opt. Express 14, 9508-9521 (2006).

86. Nieminen, T. A. et al. Optical tweezers computational toolbox. J. Opt. A: Pure Appl. Opt. 9, S196-S203 (2007).

87. Nieminen, T. A., Loke, V. L. Y., Stilgoe, A. B., Heckenberg, N. R. \& Rubinsztein-Dunlop, H. T-matrix method for modelling optical tweezers. J. Mod. Opt. 58, 528-544 (2011)

88. Saija, R., Denti, P., Borghese, F., Maragó, O. M. \& Iatì, M. A. Optical trapping calculations for metal nanoparticles: Comparison with experimental data for $\mathrm{Au}$ and Ag spheres. Opt. Express 17, 10231-10241 (2009).

89. Borghese, F., Denti, P., Saija, R., Iatì, M. A. \& Maragó, O. M. Radiation torque and force on optically trapped linear nanostructures. Phys. Rev. Lett. 100, 163903 (2008).

90. Draine, B. T. \& Flatau, P. J. Discrete-dipole approximation for scattering calculations. J. Opt. Soc. Am. A 11, 1491-1499 (1994).

91. Simpson, S. H. \& Hanna, S. Application of the discrete dipole approximation to optical trapping calculations of inhomogeneous and anisotropic particles. Opt. Express 19, 16526-16541 (2011).

92. Loke, V. L. Y., Nieminen, T. A., Heckenberg, N. R., RubinszteinDunlop, H. T-matrix calculation via discrete dipole approximation, point matching and exploiting symmetry. J. Quant. Spectrosc. Radiat. Transfer 110, 1460-1471 (2009).

93. Bareil, P. B. \& Sheng, Y. Angular and position stability of a nanorod trapped in an optical tweezers. Opt. Express 18, 26388-26398 (2010).

94. Wright, W. H., Sonek, G. J. \& Berns, M. W. Radiation trapping forces on microspheres with optical tweezers. Appl.Phys. Lett. 63, 715-717 (1993).

95. Meier, S. A. Plasmonics: Fundamentals and Applications (Springer, 2007).

96. Seol, Y., Carpenter, A. E. \& Perkins, T. T. Gold nanoparticles: enhanced optical trapping and sensitivity coupled with significant heating. Opt. Lett. 31, 2429-2431 (2006)

97. Kyrsting, A., Bendix, P. M., Stamou, D. G. \& Oddershede, L. B. Heat profiling of three-dimensionally optically trapped gold nanoparticles using vesicle cargo release. Nano Lett. 11, 888-892 (2011).

98. Yan, Z. et al. Three-dimensional optical trapping and manipulation of single silver nanowires. Nano Lett. 12, 5155-5161 (2012).

99. Zhan, Q. Trapping metallic Rayleigh particles with radial polarization. Opt. Express 12, 3377-3382 (2004)

100. Volpe, G., Singh, G. P. \& Petrov, D. Optical tweezers with cylindrical vector beams produced by optical fibers. Proc. SPIE 5514, 283-292 (2004).

101. Skelton, S. E. et al. Trapping volume control in optical tweezers using cylindrical vector beams. Opt. Lett. 38, 28-30 (2013).

102. Donato, M. G. et al. Optical trapping of nanotubes with cylindrical vector beams. Opt. Lett. 37, 3381-3383 (2012).

103. Iglesias, I. \& Sáenz, J. J. Light spin forces in optical traps: comment on "Trapping metallic Rayleigh particles with radial polarization". Opt. Express 20, 2832-2834 (2012)

104. Baffou, G. \& Quidant, R. Thermo-plasmonics: using metallic nanostructures as nano-sources of heat. Laser Photon. Rev. 7, 171-187 (2013).

105. Ma, H., Bendix, P. M. \& Oddershede, L. B. Large-scale orientation dependent heating from a single irradiated gold nanorod. Nano Lett. 12, 3954-3960 (2012).

106. Burns, M. M., Fournier, J-M. \& Golovchenko, J. A. Optical binding. Phys. Rev. Lett. 63, 1233-1236 (1989).

107. Burns, M. M., Fournier, J-M. \& Golovchenko, J. A. Optical matter: Crystallization and binding in intense optical fields. Science 249, 749-754 (1990).
108. Dholakia, K. \& Zemánek, P. Colloquium: Gripped by light: Optical binding. Rev. Mod. Phys. 82, 1767-1791 (2010).

109. Demergis, V. \& Florin, E-L. Ultrastrong optical binding of metallic nanoparticles. Nano Lett. 12, 5756-5760 (2012).

110. Yan, Z., Shah, R. A., Chado, G., Gray, S. K., Pelton, M. \& Scherer, N. F. Guiding spatial arrangements of silver nanoparticles by optical binding interactions in shaped light fields. ACS Nano 7, 1790-1802 (2013)

111. Slama-Eliau, B. N. \& Raithel, G. Three-dimensional arrays of submicron particles generated by a four-beam optical lattice. Phys. Rev. E 83, 051406 (2011).

112. Albaladejo, S., Sáenz, J. J. \& Marqués, M. I. Plasmonic nanoparticle chain in a light field: A resonant optical sail. Nano Lett. 11, 4597-4600 (2011).

113. Dapasse, F. \& Vigoureux, J. M. Optical binding force between two Rayleigh particles. J. Phys. D: Appl. Phys. 27, 914-919 (1994).

114. Bonaccorso, F. et al. Density gradient ultracentrifugation of nanotubes: Interplay of bundling and surfactants encapsulation. J. Phys. Chem. C 114, 17267-17285 (2010)

115. Neuman, K. C. \& Block, S. M. Optical trapping. Rev. Sci. Instrum. 75, 2787-2809 (2004)

116. Lee, W. M., Reece, P. J., Marchington, R. F., Metzger, N. K. \& Dholakia, K. Construction and calibration of an optical trap on a fluorescence optical microscope. Nature Protoc. 2, 3226-3238 (2007).

117. Donato, M. G. et al. Optical trapping of porous silicon nanoparticles. Nanotechnology 22, 505704 (2011).

118. Fällman, E. \& Axner, O. Design for fully steerable dual-trap optical tweezers. Appl. Opt. 36, 2107-2113 (1997).

119. Lee, S-W., Jo, G., Lee, T. \& Lee, Y-G. Controlled assembly of $\operatorname{In}_{2} \mathrm{O}_{3}$ nanowires on electronic circuits using scanning optical tweezers. Opt. Express 17, 17491-17501 (2009).

120. Mack, A. H., Trias, M. K. \& Mochrie, S. G. J. Precision optical trapping via a programmable direct-digital-synthesis-based controller for acousto-optic deflectors. Rev. Sci. Instrumen. 80, 016101 (2009).

121. Dufresne, E. R. \& Grier, D. G. Optical tweezer arrays and optical substrates created with diffractive optics. Rev. Sci. Instrumen. 69, 1974-1977 (1998).

122. Reicherter, M., Haist, T., Wagemann, E. U. \& Tiziani, H. J. Optical particle trapping with computer-generated holograms written on a liquid-crystal display. Opt. Lett. 24, 608-610 (1999).

123. Mogensen, P. C. \& Glückstad, J. Dynamic array generation and pattern formation for optical tweezers. Opt. Commun. 175, 75-81 (2000).

124. Liesener, J., Reicherter, M., Haist, T. \& Tiziani, H. Multi-functional optical tweezers using computer-generated holograms. Opt. Commun. 185, 77-82 (2000)

125. Dufresne, E. R., Spalding, G. C., Dearing, M. T., Sheets, S. A. \& Grier, D. G. Computer-generated holographic optical tweezer arrays. Rev. Sci. Instrumen. 72, 1810-1816 (2001)

126. Castelino, K., Satyanarayana, S. \& Sitti, M. Manufacturing of two and threedimensional micro/nanostructures by integrating optical tweezers with chemical assembly. Robotica 23, 435-439 (2005).

127. Novotny, L., Bian, R. X. \& Xie, X. S. Theory of nanometric optical tweezers. Phys. Rev. Lett. 79, 645-648 (1997)

128. Martin, O. J. F. \& Girard, C. Controlling and tuning strong optical field gradients at a local probe microscope tip apex. Appl. Phys. Lett. 70, 705-707 (1997).

129. Okamoto, K. \& Kawata, S. Radiation force exerted on subwavelength particles near a nanoaperture. Phys. Rev. Lett. 83, 4534-4537 (1999).

130. Garcés-Chávez, V. et al. Extended organization of colloidal microparticles by surface plasmon polariton excitation. Phys. Rev. B 73, 085417 (2006).

131. Huang, L., Maerkl, S. J. \& Martin, O. J. Integration of plasmonic trapping in a microfluidic environment. Opt. Express 17, 6018-6024 (2009).

132. Volpe, G., Volpe, G. \& Quidant, R. Fractal plasmonics: subdiffraction focusing and broadband spectral response by a Sierpinski nanocarpet. Opt. Express 19, 3612-3618 (2011)

133. Ghislain, L. P. \& Webb, W. W. Scanning-force microscope based on an optical trap. Opt. Lett. 18, 1678-1680 (1993).

134. Florin, E-L., Pralle, A., Horber, J. K. H. \& Stelzer, E. H. K. Photonic force microscope based on optical tweezers and two-photon excitation for biological applications. J. Struct. Biol. 119, 202-211 (1997).

135. Rohrbach, A., Tischer, C., Neumayer, D., Florin, E-L. \& Stelzer, E. H. K. Trapping and tracking a local probe with a photonic force microscope. Rev. Sci. Instrumen. 75, 2197-2210 (2004).

136. Pralle, A., Prummer, M., Florin, E-L., Stelzer, E. H. K. \& Horber, J. K. H. Three-dimensional high resolution particle tracking for optical tweezers by forward light scattering. Microsc. Res. Tech. 44, 378-386 (1999).

137. Tischer, C. et al. Three-dimensional thermal noise imaging. Appl. Phys. Lett. 79, 3878-3880 (2001)

138. Meiners, J-C. \& Quake, S. R. Direct measurement of hydrodynamic cross correlations between two particles in an external potential. Phys. Rev. Lett. 82, 2211-2214 (1999). 
139. Berg-Sorensen, K. \& Flyvbjerg, H. Power spectrum analysis for optical tweezers. Rev. Sci. Instrumen. 75, 594-612 (2004).

140. Brettschneider, T., Volpe, G., Helden, L., Wehr, J. \& Bechinger, C. Force measurement in the presence of Brownian noise: Equilibrium distribution method versus drift method. Phys. Rev. E 83, 041113 (2011).

141. Kress, H., Stelzer, E. H. K. \& Rohrbach, A. Tilt angle dependent three-dimensional-position detection of a trapped cylindrical particle in a focused laser beam. Appl. Phys. Lett. 84, 4271-4273 (2004).

142. Ito, S., Yoshikawa, H. \& Masuhara, H. Optical patterning and photochemical fixation of polymer nanoparticles on glass substrates. Appl. Phys. Lett. 78, 2566-2568 (2001).

143. Guffey, M. J. \& Scherer, N. F. All-optical patterning of Au nanoparticles on surfaces using optical traps. Nano Lett. 10, 4302-4308 (2010).

144. Nedev, S., Urban, A. S., Lutich, A. A. \& Feldmann, J. Optical force stamping lithography. Nano Lett. 11, 5066-5070 (2011).

145. Woerdemann, M. et al. Dynamic and reversible organization of Zeolite L crystals induced by holographic optical tweezers. Adv. Mater. 22, 4176-4179 (2010)

146. McLeod, E. \& Arnold, C. B. Subwavelength direct-write nanopatterning using optically trapped microspheres. Nature Nanotech. 3, 413-417 (2008).

147. Tsai, Y-C., Leitz, K-H., Fardel, R., Otto, A., Schmidt, M. \& Arnold, C. B. Parallel optical trap assisted nanopatterning on rough surfaces. Nanotechnology 23, 165304 (2012)

148. Prikulis, J. et al. Optical spectroscopy of single trapped metal nanoparticles in solution. Nano Lett. 4, 115-118 (2004).

149. Ohlinger, A., Nedev, S., Lutich, A. A. \& Feldman, J. Optothermal escape of plasmonically coupled silver nanoparticles from a three dimensional optical trap. Nano Lett. 11, 1770-1774 (2011).

150. Xie, C. G. et al. Near-infrared raman spectroscopy of single optically trapped biological cells. Opt. Lett. 27, 249-251 (2002).

151. Huang, Y., Duan, X. \& Lieber, C. M. Nanowires for integrated multicolor nanophotonics. Small 1, 142-147 (2005).

152. O'Connell, M. J. et al. Band gap fluorescence from individual single-walled carbon nanotubes. Science 297, 593-596 (2002).

153. Tan, P. et al. Photoluminescence spectroscopy of carbon nanotube bundles: Evidence for exciton energy transfer. Phys. Rev. Lett. 99, 137402 (2007).

154. Hertel, T. et al. Spectroscopy of single-and double-wall carbon nanotubes in different environments. Nano Lett. 5, 511-514 (2005).

155. Ferrari, A. C. et al. Raman spectrum of graphene and graphene layers. Phys. Rev. Lett. 97, 187401 (2007).

156. Ferrari, A. C. \& Basko, D. M. Raman spectroscopy as a versatile tool for studying the properties of graphene. Nature Nanotech. 8, 235-246 (2013).

157. Aizpurua, J. et al. Optical properties of coupled metallic nanorods for fieldenhanced spectroscopy. Phys. Rev. B 71, 235420 (2005).
158. Prodan, E. et al. A hybridization model for the plasmon response of complex nanostructures. Science 302, 419-422 (2003).

159. Kneipp, K., Moskovits, M. \& Kneipp, H. Surface-Enhanced Raman Scattering (Springer, 2006).

160. Willets, K. A. \& Van Duyne, R. P. Localized surface plasmon resonance spectroscopy and sensing. Annu. Rev. Phys. Chem. 58, 267-297 (2007).

161. McDougall, C., Stevenson, D. J., Brown, C. T. A., Gunn-Moore, F. \& Dholakia, K. Targeted optical injection of gold nanoparticles into single mammalian cells. J. Biophotonics 2, 736-743 (2009).

162. Stevenson, D. et al. Femtosecond optical transfection of cells: viability and efficiency. Opt. Express 14, 7125-7133 (2006).

163. Cleland, A. Optomechanics: Photons refrigerating phonons. Nature Phys. 5, 458-460 (2009).

164. Kane, B. E. Levitated spinning graphene flakes in an electric quadrupole ion trap. Phys. Rev. B 82, 115441 (2010).

165. Hernandez, Y. et al. High-yield production of graphene by liquid phase exfoliation of graphite. Nature Nanotech. 3, 563-568 (2008).

166. Li, T., Kheifets, S. \& Raizen, M. G. Millikelvin cooling of an optically trapped microsphere in vacuum. Nature Phys. 7, 527-530 (2011).

167. Ridolfo, A. et al. Fano-doppler laser cooling of hybrid nanostructures. ACS Nano 5, 7354-7361 (2011).

168. Juan, M. L., Righini, M. \& Quidant, R. Plasmon nano-optical tweezers. Nature Photon. 5, 349-356 (2011).

169. Radisavljevic, B., Radenovic, A., Brivio, J., Giacometti, V. \& Kis, A. Single-layer $\mathrm{MoS}_{2}$ transistors. Nature Nanotech. 6, 147-150 (2011).

\section{Acknowledgements}

We thank F. Bonaccorso, B. Fazio, C. J. Foot, M. G. Donato, M. A. Iatì, A. Irrera, R. Saija, S. Savasta and G. Volpe for discussions. We acknowledge funding from FP7-HEALTH-F5-2009-241818 NANOANTENNA, MPNS COST Action 1205 "Advances in Optofluidics: Integration of Optical Control and Photonics with Microfluidics", the Leverhulme Trust, the Scientific and Technological Research Council of Turkey (TUBITAK) under Grants 111T758 and 112T235, Marie Curie Career Integration Grant (MC-CIG) under Grant PCIG11 GA-2012-321726, COST Action IC1208, the Royal Society, the European Research Council Grant NANOPOTS, EU Grants RODIN, MEM4WIN, and Graphene Flagship, EPSRC grants EP/K01711X/1, EP/K017144/1, EP/G042357/1 and Nokia Research Centre, Cambridge.

\section{Additional information}

Reprints and permissions information is available online at www.nature.com/reprints. Correspondence should be addressed to O.M.M. and A.C.F.

\section{Competing financial interests}

The authors declare no competing financial interests. 